\title{
Elucidating Vehicle Lateral Dynamics Using a Bifurcation Analysis
}

\author{
Der-Cherng Liaw, Senior Member, IEEE, Hsin-Han Chiang, Member, IEEE, and Tsu-Tian Lee, Fellow, IEEE
}

\begin{abstract}
Issues of stability and bifurcation phenomena in vehicle lateral dynamics are presented. Based on the assumption of constant driving speed, a second-order nonlinear lateral dynamics model is obtained. Local stability and existence conditions for saddle-node bifurcation appearing in vehicle dynamics with respect to the variations in front wheel steering angle are then derived via system linearization and local bifurcation analysis. Bifurcation phenomena occurring in vehicle lateral dynamics might result in spin and/or system instability. A perturbation method is employed to solve for an approximation of system equilibrium near the zero value of the front wheel steering angle, which reveals the relationship between sideslip angle and the applied front wheel angle. Numerical simulations from an example model demonstrate the theoretical results.
\end{abstract}

Index Terms-Bifurcation analysis, perturbation method, vehicle's lateral dynamics.

\section{INTRODUCTION}

$\mathbf{I}$ N RECENT years, the study of vehicle lateral dynamics has attracted considerable attention (e.g., [1]-[9]). One of the major concerns in vehicle dynamics is safety. Due to the large number of traffic accidents occurring daily, the link between the nonlinear behavior of vehicle dynamics and the applied front wheel steering angle becomes a very important issue. Among the existing studies, the sliding mode approach has been used to design robust control laws for providing system stability with respect to large variations in system parameters such as axial velocity, vehicle mass, and the contact force between tire and road surface [3]. A 5-degree-of-freedom (DOF) vehicle model was used in [7] to design an extended Kalman filter for estimating the historic data of vehicle motion and tire force. Based on a linear model of vehicle lateral dynamics, linear control laws have been proposed in [4] and [5].

Bifurcation theory and its corresponding analytical techniques have been recently well exploited (e.g., [10]-[14]). These methods have been successfully applied to the study and control of several engineering systems such as tethered satellite systems, jet engine compressors, longitudinal flight dynamics,

Manuscript received October 24, 2005; revised July 10, 2006, September 19, 2006, September 20, 2006, and September 29, 2006. This work was supported by the Program for Promoting Academic Excellence of Universities under Grant 91X104 EX-91-E-FA06-4-4. The Associate Editor for this paper was R. W. Goudy.

D.-C. Liaw and H.-H. Chiang are with the Department of Electrical and Control Engineering, National Chiao Tung University, Hsinchu 300, Taiwan, R.O.C. (e-mail: 1dc@cn.nctu.edu.tw; hsinhan.ece90g@nctu.edu.tw).

T.-T. Lee is with the National Taipei University of Technology, Taipei 106, Taiwan, R.O.C. (e-mail: ttlee@ntut.edu.tw).

Color versions of one or more of the figures in this paper are available online at http://ieeexplore.ieee.org.

Digital Object Identifier 10.1109/TITS.2006.888598

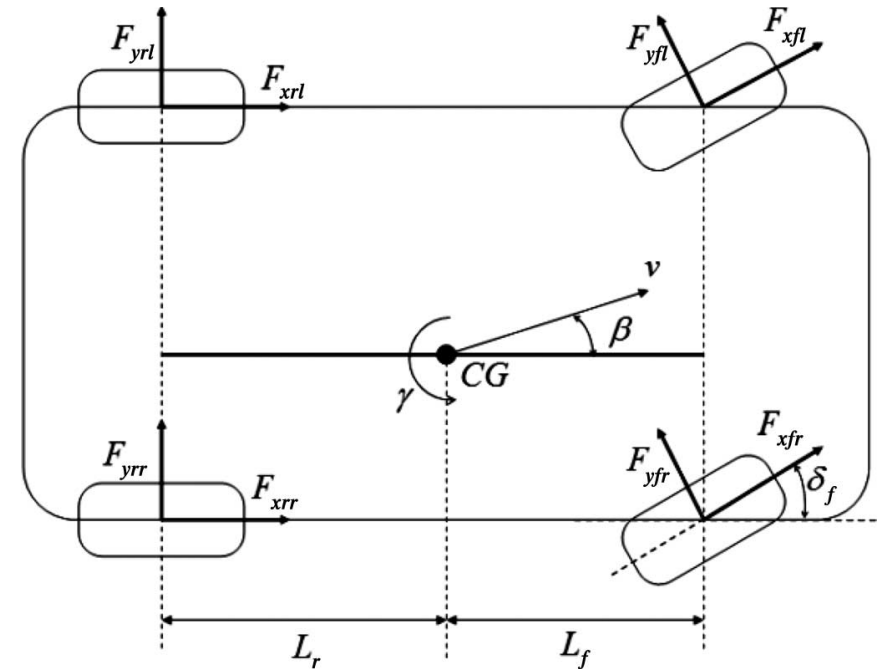

Fig. 1. Front-steering vehicle model.

and power systems (e.g., [15]-[18]). Although saddle-node bifurcation in vehicle dynamics was observed in [6] to link with system instability via a numerical example, no theoretical analysis of possible bifurcation phenomena has been studied yet.

Instead of using a numerical approach, the main goal of this paper is to provide an analytical study of vehicle lateral dynamics. Based on previous successful applications to the analytical study of longitudinal flight dynamics, tethered satellite systems and jet engine compressors by Liaw et al. [15]-[18], the existences and corresponding stability conditions of system equilibrium will be analytically discussed in this paper.

This paper is organized as follows. Nonlinear dynamics of a vehicle system is recalled in Section II. It is followed by the analysis of existences and the corresponding stability conditions for system equilibrium. Analytical formulas will then be applied to the detection of possible occurrences of local bifurcation phenomena and the construction of corresponding existence conditions. Numerical studies with CarSim software [21] are also given in Section IV to demonstrate the analytical results.

\section{NONLINEAR Vehicle DyNAmics}

Consider the vehicle's steering dynamics as depicted in Fig. 1 (e.g., [19]). Here, we have front-wheel angle $\delta_{\mathrm{f}}$ as system input and both sideslip angle $\beta$ and yaw rate $\gamma$ as two system outputs for the steering characteristics. In addition, $L_{\mathrm{f}}$ denotes the distance between the center of gravity (CG) and front-wheel axle, and $L_{\mathrm{r}}$ is the distance between CG and rear-wheel axle, respectively. $F_{y \mathrm{fl}}$ and $F_{y \mathrm{rl}}$ are the cornering forces of the left front and 


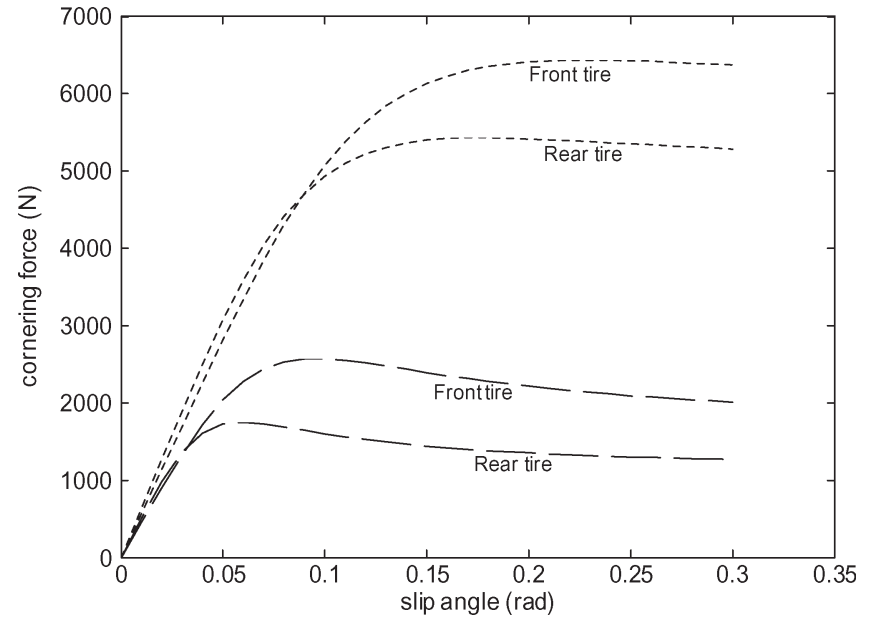

Fig. 2. Cornering characteristics of front and rear tires. Dotted line: highfriction road, and dashed line: low-friction road.

left rear tires, while $F_{y \text { fr }}$ and $F_{y \text { rr }}$ are the cornering forces for the right front and right rear tires. $F_{x f l}$ and $F_{x \mathrm{rl}}$ are the traction forces for the left front and left rear tires, while $F_{x \mathrm{fr}}$ and $F_{x \mathrm{rr}}$ are the traction forces for the right front and right rear tires.

We assume the vehicle body is symmetric about the longitudinal plane. Let $F_{y \mathrm{f}}=F_{y \mathrm{fl}}+F_{y \mathrm{fr}}, F_{y \mathrm{r}}=F_{y \mathrm{rl}}+F_{y \mathrm{rr}}, F_{x \mathrm{f}}=$ $F_{x \mathrm{fl}}+F_{x \mathrm{fr}}$, and $F_{x \mathrm{r}}=F_{x \mathrm{rl}}+F_{x \mathrm{rr}}$. The basic equations of motion for steering dynamics with roll motion neglected were derived (e.g., [19]) and given as

$$
\begin{aligned}
& m(\dot{\nu}-\nu \beta \gamma)=F_{x \mathrm{f}}+F_{x \mathrm{r}}-F_{y \mathrm{f}} \sin \delta_{\mathrm{f}} \\
& m \nu \cdot(\dot{\beta}+\gamma)=F_{y \mathrm{f}}+F_{y \mathrm{r}}+F_{x \mathrm{f}} \sin \delta_{\mathrm{f}}
\end{aligned}
$$

and

$$
I_{z} \dot{\gamma}=\left(L_{\mathrm{f}} F_{y \mathrm{f}}-L_{\mathrm{r}} F_{y \mathrm{r}}\right) \cos \beta+L_{\mathrm{f}} F_{x \mathrm{f}} \sin \delta_{\mathrm{f}}
$$

where $m$ is the mass of the vehicle, $I_{z}$ is the yaw moment around $z$-axis, and $\nu$ is the longitudinal velocity.

In this paper, we focus on the characteristic analysis of lateral dynamics by assuming $F_{x \mathrm{f}}=0$, and the vehicle is not accelerating or decelerating along the longitudinal direction, i.e., $\dot{\nu}=0$. Thus, (1) can then be neglected in this analysis. The steering dynamics for constant speed $\nu$ can then be reduced to a second-order model as given by

$$
\begin{aligned}
& \dot{\beta}=\frac{1}{m \nu}\left\{F_{y \mathrm{f}}+F_{y \mathrm{r}}\right\}-\gamma \\
& \dot{\gamma}=\frac{1}{I_{z}}\left\{L_{\mathrm{f}} F_{y \mathrm{f}}-L_{\mathrm{r}} F_{y \mathrm{r}}\right\} \cos \beta
\end{aligned}
$$

where $F_{y \mathrm{f}}$ is a function of $\beta, \gamma$, and $\delta_{\mathrm{f}}$, and $F_{y \mathrm{r}}$ is a function of $\beta$ and $\gamma$ only. Examples of $F_{y \mathrm{f}}$ and $F_{y \mathrm{r}}$ are given in (21) and (22).

\section{Stability AND BifUrcation AnAlysis}

Here, we will study the vehicle steering dynamics by using the second-order model given by (4) and (5) instead of (1)-(3). Details are given as follows.
TABLE I

VeHICLE PARAMETERS AND VALUES

\begin{tabular}{ll}
\hline \hline Symbol and description & Values \\
\hline$m:$ vehicle mass & $1500 \mathrm{~kg}$ \\
$I_{z}:$ yaw moment & $3000 \mathrm{~kg} \cdot \mathrm{m}^{2}$ \\
$v:$ velocity & $10 \sim 40 \mathrm{~m} / \mathrm{s}$ \\
$L_{f}$ & $1.2 \mathrm{~m}$ \\
$L_{r}$ & $1.3 \mathrm{~m}$ \\
\hline \hline
\end{tabular}

TABLE II

COEFFicients OF MAGic Formula

\begin{tabular}{lll}
\hline \hline Symbol & High friction road & Low friction road \\
\hline$B_{f}, B_{r}$ & $6.7651,9.0051$ & $11.275,18.631$ \\
$C_{f}, C_{r}$ & $1.3,1.3$ & $1.56,1.56$ \\
$D_{f}, D_{r}$ & $-6436.8,-5430$ & $-2574.7,-1749.7$ \\
$E_{f}, E_{r}$ & $-1.999,-1.7908$ & $-1.999,-1.7908$ \\
\hline \hline
\end{tabular}

\section{A. Stability Analysis}

Define $x^{0}=\left(\beta^{0}, \gamma^{0}\right)^{\mathrm{T}}$ as an equilibrium point of system (4), (5) for a given $\delta_{\mathrm{f}}=\delta_{\mathrm{f}}^{0}$. We then have

$$
F_{y \mathrm{f}}\left(\beta^{0}, \gamma^{0}, \delta_{\mathrm{f}}^{0}\right)+F_{y \mathrm{r}}\left(\beta^{0}, \gamma^{0}, \delta_{\mathrm{f}}^{0}\right)=\gamma^{0} \cdot m \nu
$$

and

$$
L_{\mathrm{f}} F_{y \mathrm{f}}\left(\beta^{0}, \gamma^{0}, \delta_{\mathrm{f}}^{0}\right)=L_{\mathrm{r}} F_{y \mathrm{r}}\left(\beta^{0}, \gamma^{0}, \delta_{\mathrm{f}}^{0}\right)
$$

or

$$
\cos \beta^{0}=0
$$

Consider the condition as given in (8), we have $\beta^{0}=n \pi+$ $\pi / 2$ for $n=0,1,2, \ldots$. It is clear that this condition cannot be achieved for a vehicle. Thus, the equilibrium point $x^{0}$ should satisfy the two conditions given in (6) and (7) only.

Let $x=[\beta, \gamma]^{\mathrm{T}}$ and $\tilde{x}=x-x^{0}$. Taking the linearization of system (4), (5) at $x=x^{0}$, we have

$$
\dot{\tilde{x}}=A \tilde{x}
$$

where

$$
A=\left[\begin{array}{ll}
a_{1} & a_{2} \\
a_{3} & a_{4}
\end{array}\right]
$$

with

$a_{1}=\frac{1}{m \nu} \frac{\partial}{\partial \beta}\left(F_{y \mathrm{f}}+F_{y \mathrm{r}}\right)\left(\beta^{0}, \gamma^{0}, \delta_{\mathrm{f}}^{0}\right)$

$a_{2}=\frac{1}{m \nu} \frac{\partial}{\partial r}\left(F_{y \mathrm{f}}+F_{y \mathrm{r}}\right)\left(\beta^{0}, \gamma^{0}, \delta_{\mathrm{f}}^{0}\right)-1$

$a_{3}=\frac{1}{I_{z}}\left(L_{\mathrm{f}} \frac{\partial F_{y \mathrm{f}}\left(\beta^{0}, \gamma^{0}, \delta_{\mathrm{f}}^{0}\right)}{\partial \beta}-L_{\mathrm{r}} \frac{\partial F_{y \mathrm{r}}\left(\beta^{0}, \gamma^{0}, \delta_{\mathrm{f}}^{0}\right)}{\partial \beta}\right) \cos \beta^{0}$

and

$a_{4}=\frac{1}{I_{z}}\left(L_{\mathrm{f}} \frac{\partial F_{y \mathrm{f}}\left(\beta^{0}, \gamma^{0}, \delta_{\mathrm{f}}^{0}\right)}{\partial r}-L_{\mathrm{r}} \frac{\partial F_{y \mathrm{r}}\left(\beta^{0}, \gamma^{0}, \delta_{\mathrm{f}}^{0}\right)}{\partial r}\right) \cos \beta^{0}$. 


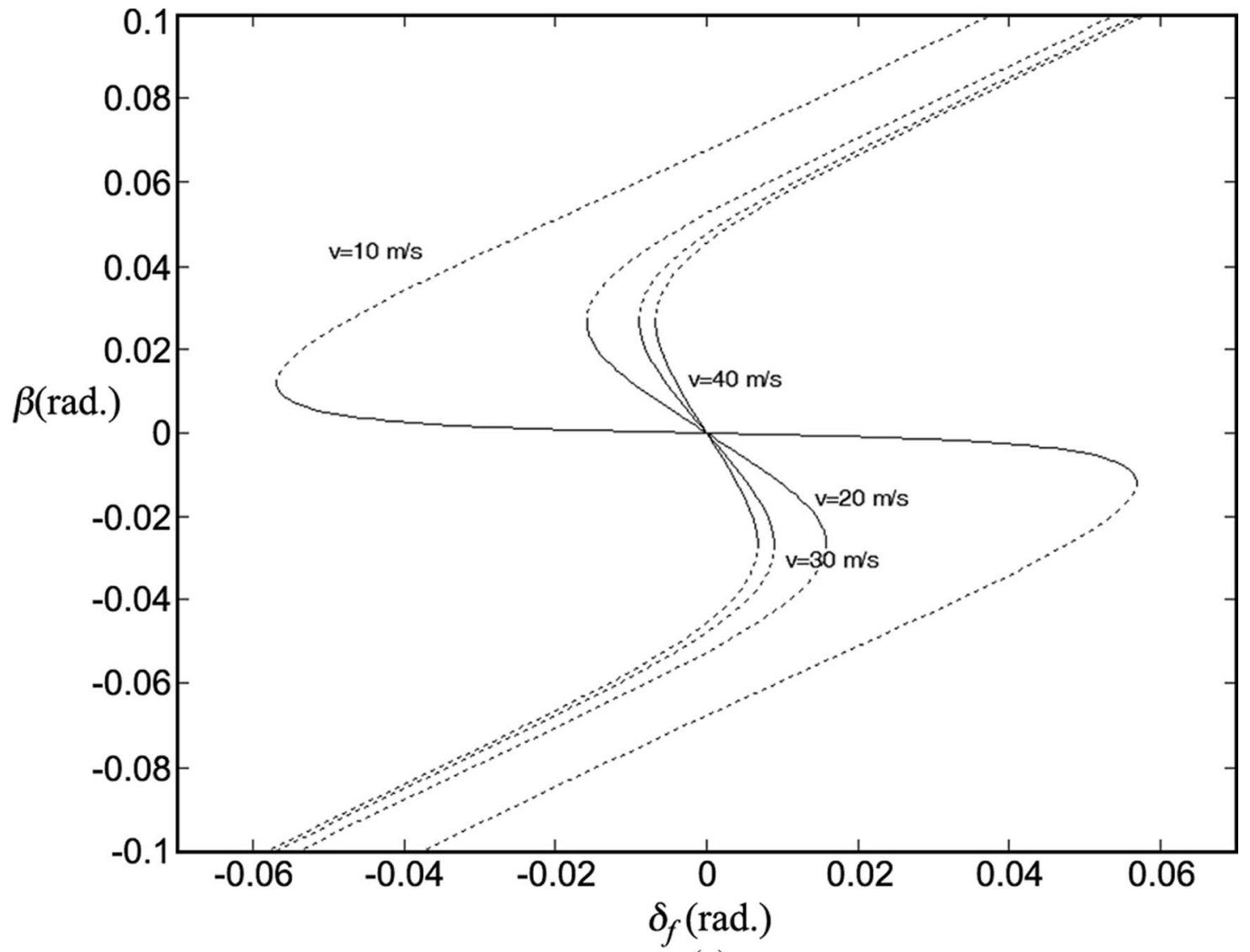

(a)

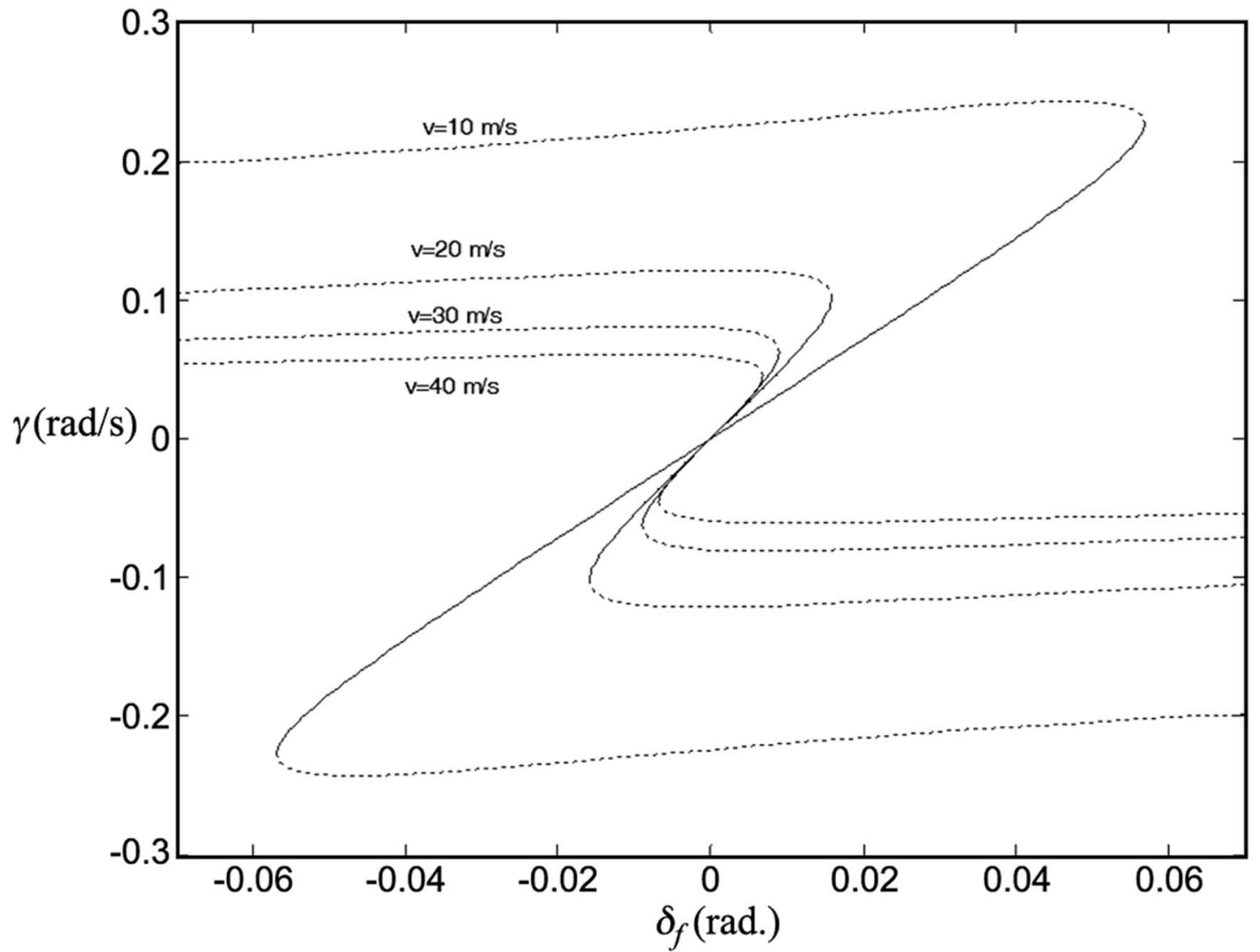

(b)

Fig. 3. Bifurcation diagram with respect to different setting of $\nu$. (a) Side-slip angle versus front-wheel angle. (b) Yaw rate versus front-wheel angle. 


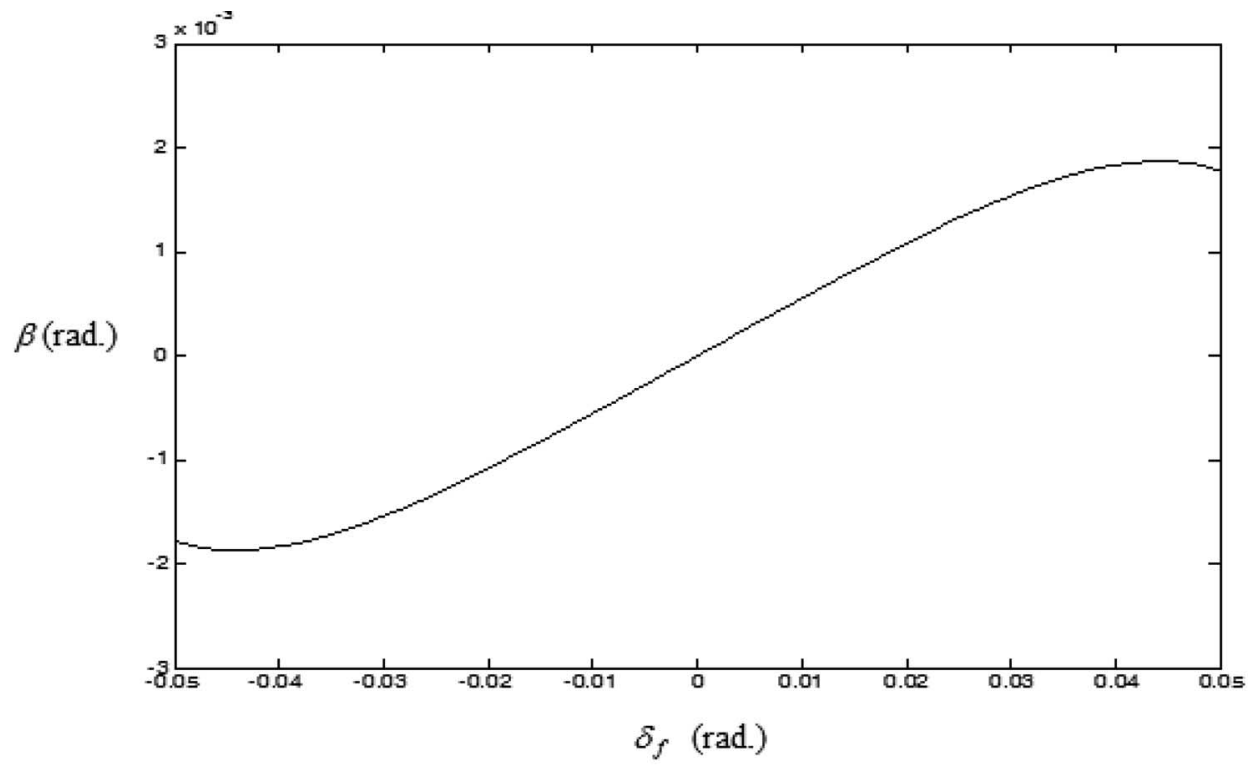

(a)

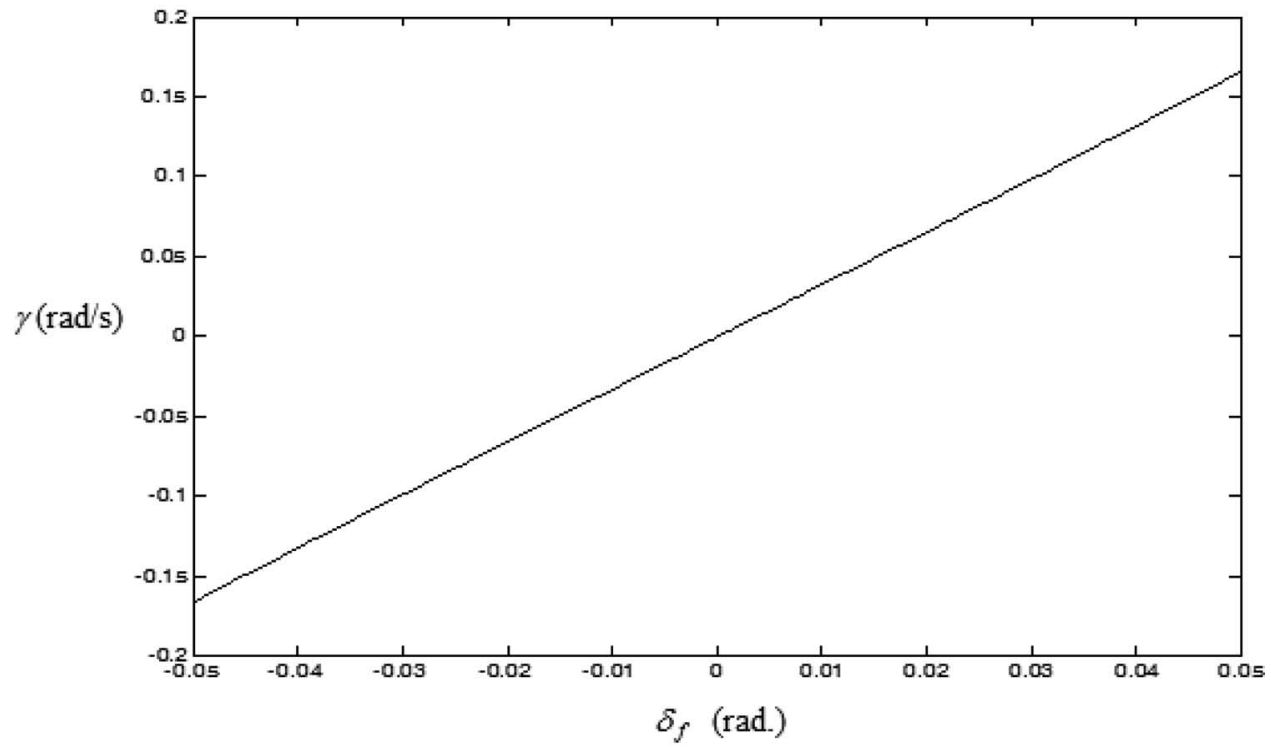

(b)

Fig. 4. Equilibrium point valued at $\nu=9 \mathrm{~m} / \mathrm{s}$. (a) Side-slip angle versus front-wheel angle. (b) Yaw rate versus front-wheel angle.

By applying the Routh-Hurwitz stability criterion, we then have the following stability results.

Lemma 1: The equilibrium point $x^{0}$ of system (4), (5) is asymptotically stable if $a_{1}+a_{4}<0$ and $a_{1} a_{4}-a_{2} a_{3}>0$. Here, the $a_{i}$ s are given in (11)-(14). Moreover, the equilibrium point $x^{0}$ is unstable if $a_{1}+a_{4}>0$ and $a_{1} a_{4}-a_{2} a_{3}<0$.

Observation 1: It is known from the so-called "Magic formula" that both values of $a_{1}$ and $a_{4}$ will generally be negative. An example is given in Section III. Thus, the stability condition in Lemma 1 can then be reduced to where the equilibrium point $x^{0}$ of system (4), (5) will be stable if $a_{1} a_{4}-a_{2} a_{3}>0$ and unstable if $a_{1} a_{4}-a_{2} a_{3}<0$.

From Observation 1 above, it is clear that the system linearization of (4), (5) at $x^{0}$ will in general not have a pair of pure imaginary eigenvalues but might have zero eigenvalue. That means the lateral dynamics of a vehicle system will not
TABLE III

SADDlE-Node BIFURCATION FOR DifFERENT SPEEDS

\begin{tabular}{ccccc}
\hline \hline$\nu(\mathrm{m} / \mathrm{s})$ & Label & $\delta_{f}(\mathrm{rad})$. & $\beta^{0}(\mathrm{rad})$. & $\gamma^{0}(\mathrm{rad} . / \mathrm{s})$ \\
\hline \multirow{2}{*}{10} & SNBP1 & -0.0569 & 0.0120 & -0.2275 \\
& SNBP2 & 0.0569 & -0.0120 & 0.2275 \\
\hline \multirow{2}{*}{20} & SNBP1 & -0.0158 & 0.0267 & -0.1017 \\
& SNBP2 & 0.0158 & -0.0267 & 0.1017 \\
\hline \multirow{2}{*}{30} & SNBP1 & -0.0089 & 0.0272 & -0.0631 \\
& SNBP2 & 0.0089 & -0.0272 & 0.0631 \\
\hline \multirow{2}{*}{40} & SNBP1 & -0.0067 & 0.0267 & -0.0454 \\
& SNBP2 & 0.0067 & -0.0267 & 0.0454 \\
\hline \hline
\end{tabular}

undergo Hopf bifurcation (e.g., [12]-[14]). However, it might have chance for the appearance of stationary bifurcation at some $\delta_{\mathrm{f}}=\delta_{\mathrm{f}}^{0}$ such that $a_{1} a_{4}-a_{2} a_{3}=0$. 


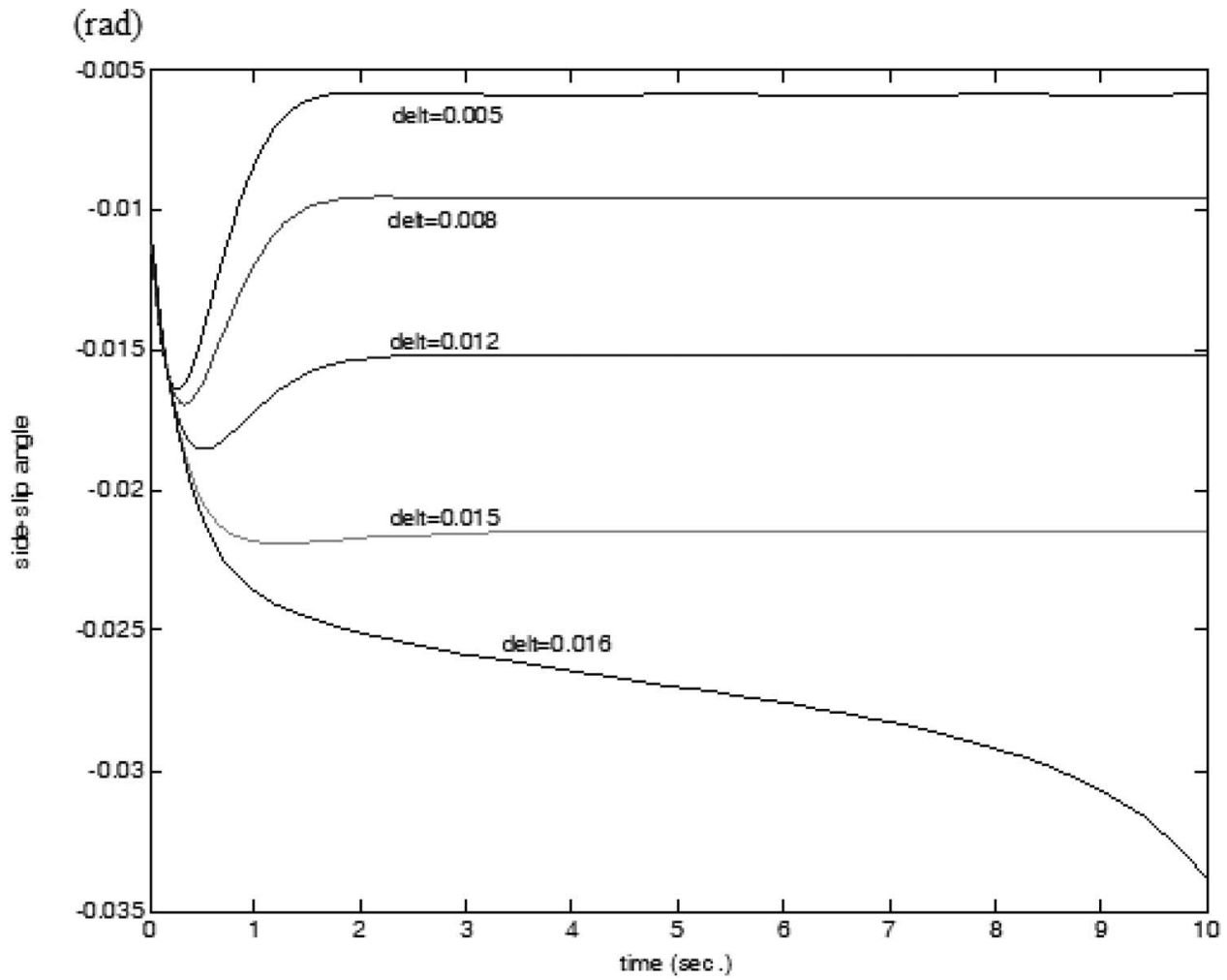

(a)

$(\mathrm{rad} / \mathrm{s})$

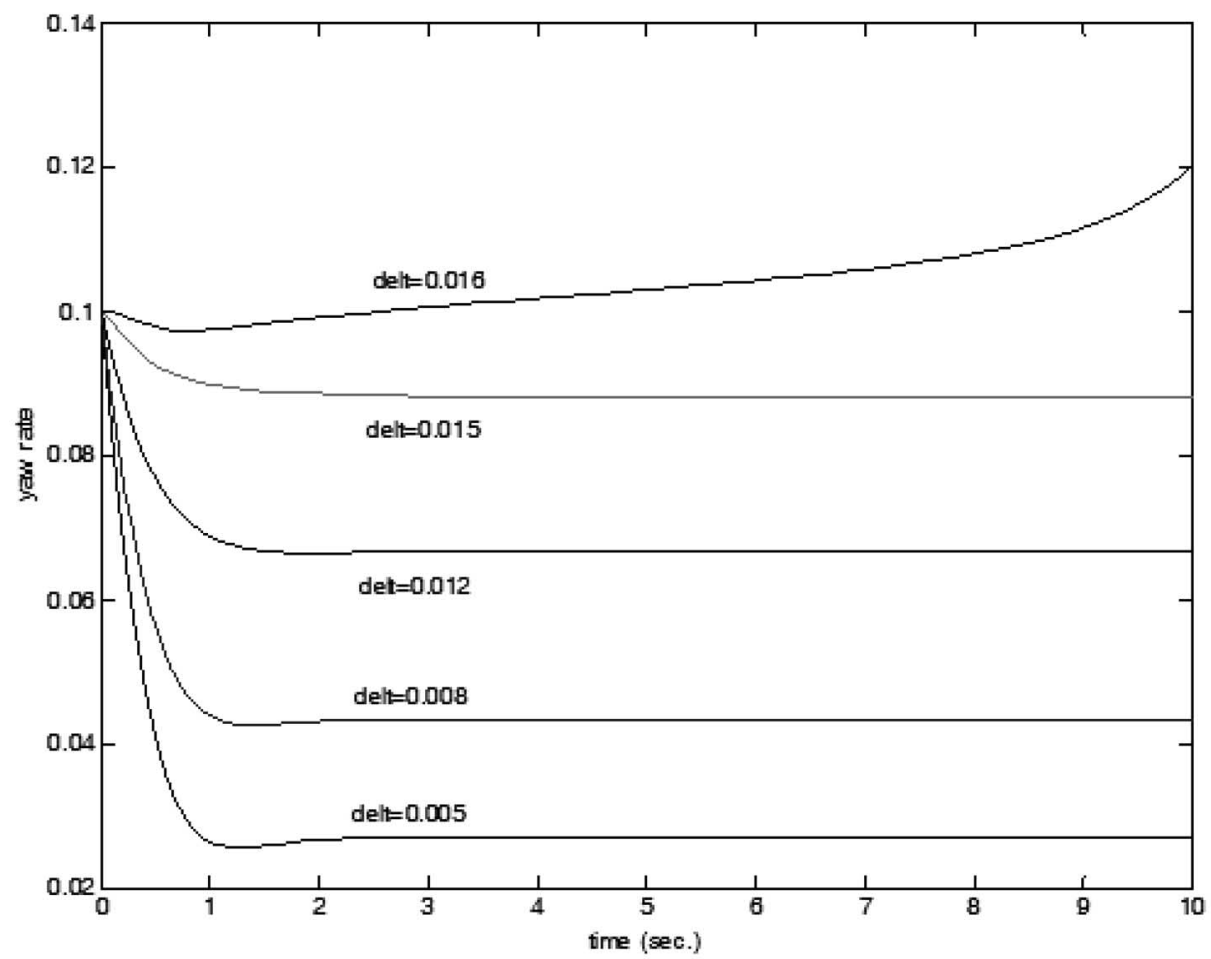

(b)

Fig. 5. Time responses with initials: $\beta=-0.01 \mathrm{rad}$ and $\gamma=0.1 \mathrm{rad} / \mathrm{s}$. (a) Side-slip angle $\beta$ versus time. (b) Yaw rate $\gamma$ versus time.

\section{B. Local Bifurcations}

In the following, we will discuss the possibility of having a stationary bifurcation (e.g., [11] and [16]) for system (4), (5). Let $x^{0}$ be the equilibrium point such that $a_{1} a_{4}-a_{2} a_{3}=0$ with $a_{1}<0$ and $a_{4}<0$. This implies that the linearization of system (4), (5) at $x^{0}$ possesses one zero eigenvalue and a stable eigenvalue $\lambda=a_{1}+a_{4}<0$. In order to study the possible occurrence of stationary bifurcation for system (4), (5), 


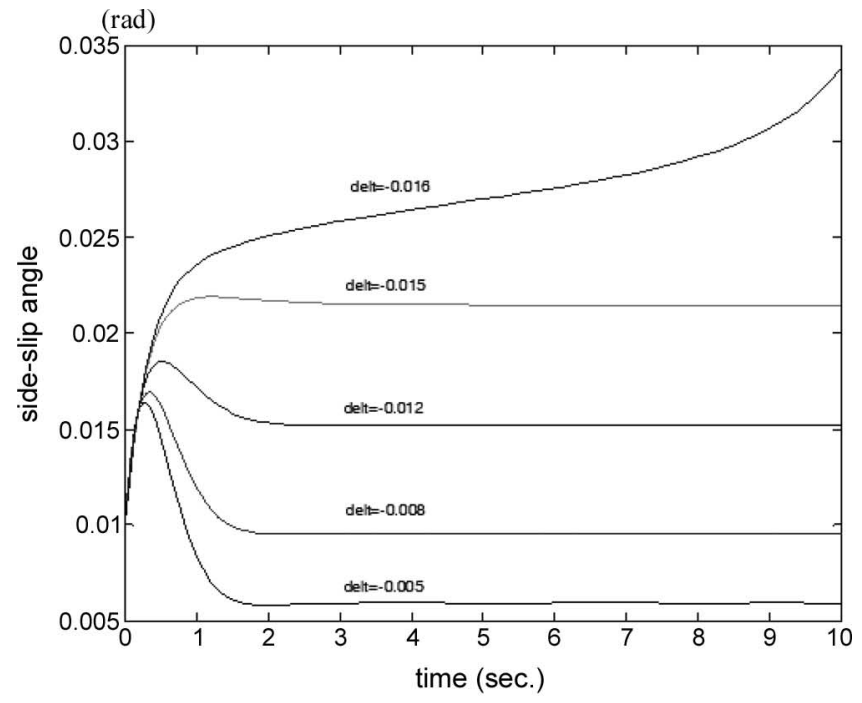

(a)

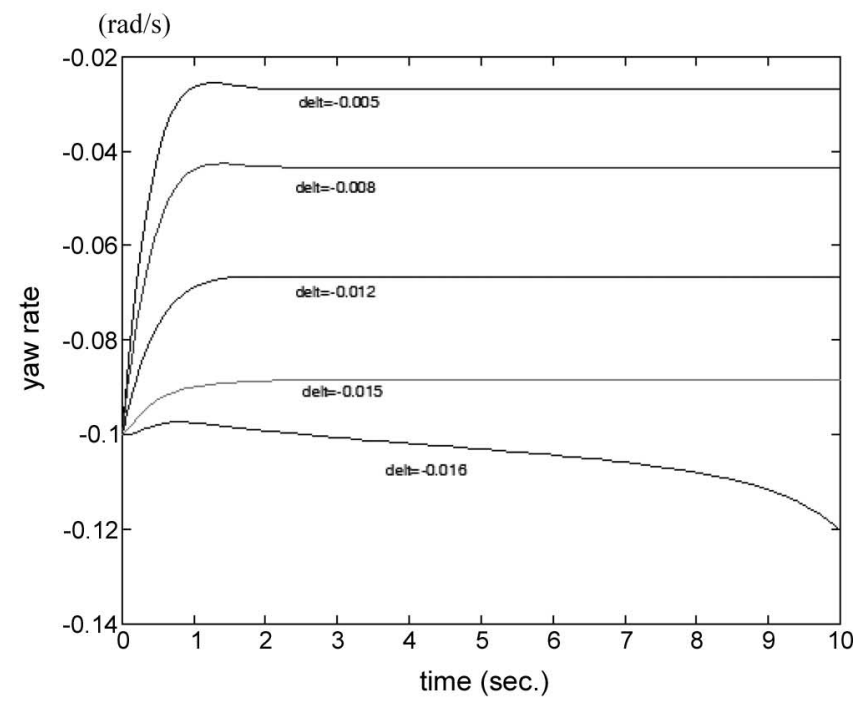

(b)

Fig. 6. Time responses with initials: $\beta=0.01$ and $\gamma=-0.1$. (a) Side-slip angle $\beta$ versus time. (b) Yaw rate $\gamma$ versus time.

we recall the result regarding the existence condition of the so-called "saddle-node bifurcation" from (e.g., [10], [12], and [13]), as presented below.

Consider a class of nonlinear system with the approximation up to the second order in its state $x$ as given by

$$
\dot{x}=L_{0} x+Q_{0}(x, x)+g \mu .
$$

Here, the Jacobian matrix $L_{0}$ possesses one zero eigenvalue with remaining eigenvalues lying in the open left-half of the complex plane. The variable $\mu$ denotes the system parameter, and $Q_{0}(x, x)$ denotes the quadratic term in $x$. Moreover, assume $x=0$ is an equilibrium point of system (15) at $\mu=0$.

We recall the following lemma (e.g., [10]) regarding the existence conditions of saddle-node bifurcation.

Lemma 2: The equilibrium point $x=0$ of system (15) will undergo saddle-node bifurcation from the origin at $\mu=0$ if

1) $l g \neq 0$

2) $l Q_{0}(r, r) \neq 0$.
TABLE IV

EXISTENCE CONDITION OF THEOREM 1

\begin{tabular}{l|l}
\hline \hline$a_{1}=-2.17032$ & $q_{11}=-69.0817$ \\
\hline$a_{2}=-1.01782$ & $q_{12}=2.08401$ \\
\hline$a_{3}=-3.56284$ & $q_{13}=-0.279671$ \\
\hline$a_{4}=-1.67372$ & $q_{21}=416.744$ \\
\hline$g_{1}=1.27114$ & $q_{22}=-56.0221$ \\
\hline$g_{2}=15.2482$ & $q_{23}=1.90615$ \\
\hline$a_{1} a_{4}-a_{2} a_{3} \approx 0$, with $a_{1}, a_{4}<0$ \\
\hline$a_{4} g_{1}-a_{2} g_{2} \approx 13.3924 \neq 0$ \\
\hline$a_{4} q_{11}-a_{3} q_{12}+\frac{a_{3}{ }^{2}}{a_{4}} q_{13}-a_{2} q_{22}-\frac{a_{1}{ }^{2}}{a_{2}} q_{23} \approx 679.75 \neq 0$ \\
\hline
\end{tabular}

$\delta_{f}(\mathrm{rad})$

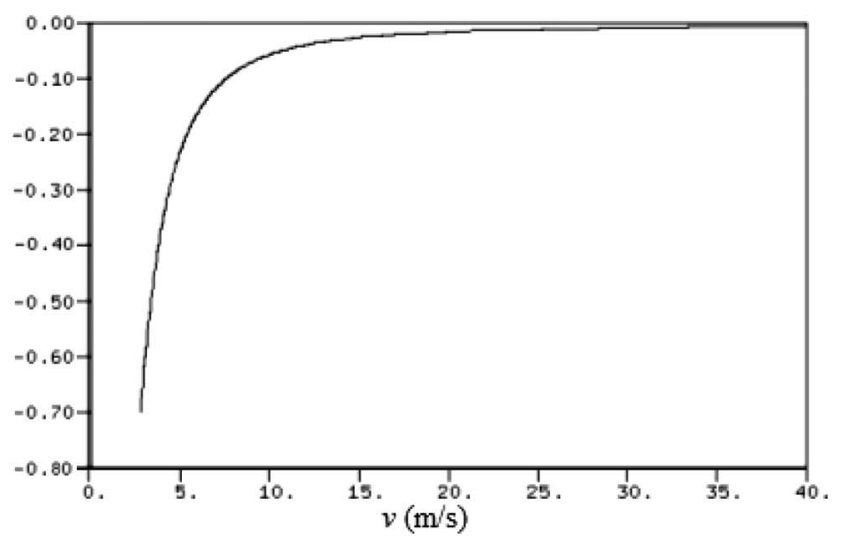

(a)

$\delta_{f}(\mathrm{rad})$

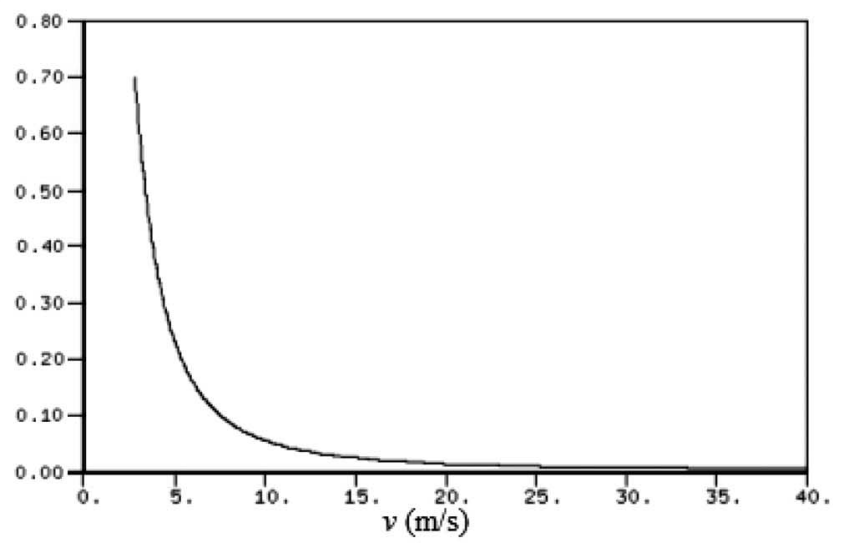

(b)

Fig. 7. Saddle-node bifurcation point in $\left(\delta_{\mathrm{f}}-\nu\right)$ space.

Here, $l$ and $r$ denote the left and right eigenvectors corresponding to the zero eigenvalues of $L_{0}$, respectively, with $l r=1$.

Now, we apply Lemma 2 to the study of local bifurcation for system (4), (5). Define $x^{0}$ as the equilibrium point of system (4), (5) with $a_{1} a_{4}=a_{2} a_{3}$. Let $\tilde{x}=x-x^{0} \triangleq\left(x_{1}, x_{2}\right)^{\mathrm{T}}$ and $\mu=\delta_{\mathrm{f}}-\delta_{\mathrm{f}}^{0}$. Taking the Taylor series expansion of the system (4), (5) at $\left(x^{0}, \delta_{\mathrm{f}}^{0}\right)$, we then have

$$
\dot{\tilde{\mathrm{x}}}=A \tilde{x}+g \mu+Q(\tilde{x}, \tilde{x})+O(2)
$$




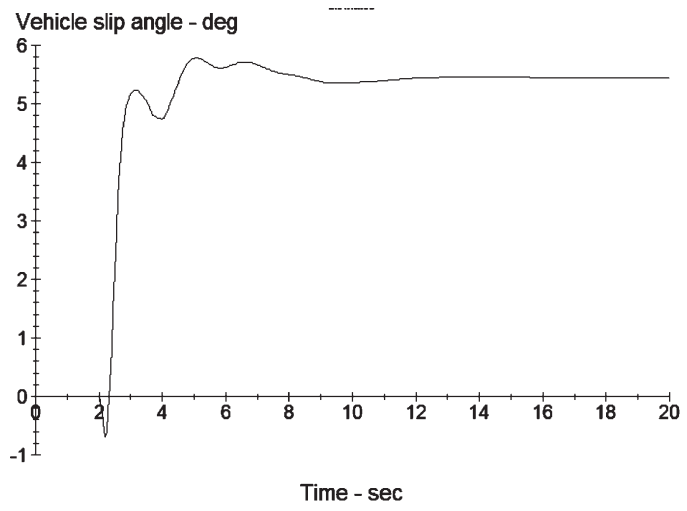

(a)

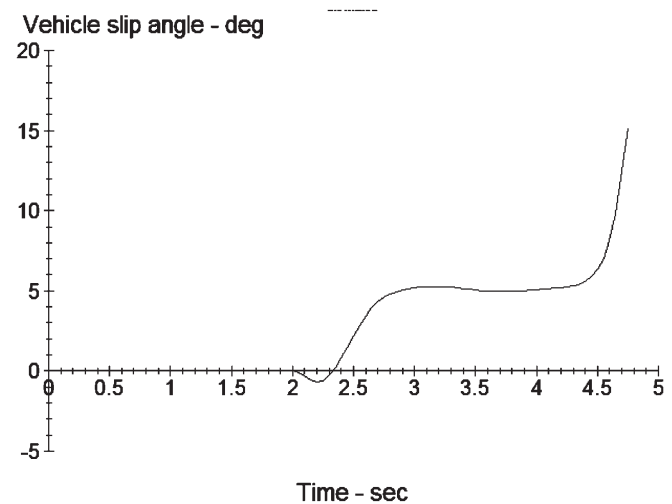

(c)

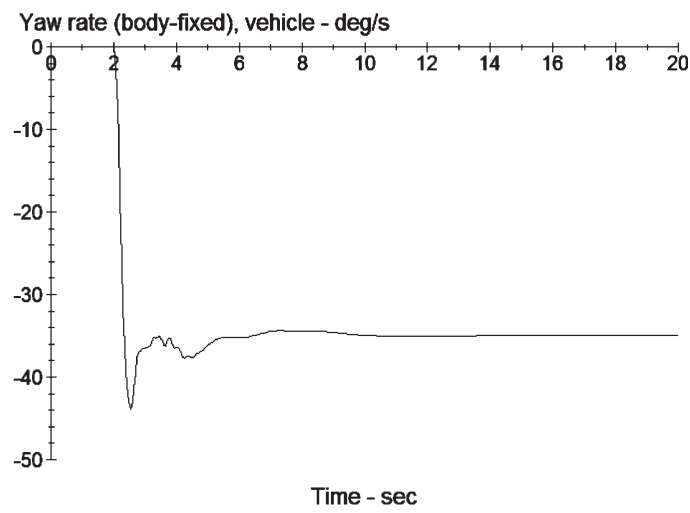

(b)

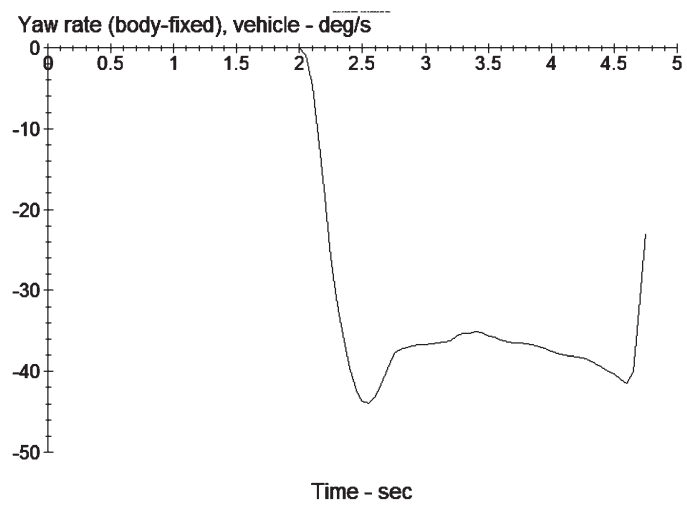

(d)

Fig. 8. Vehicle states (a) slip angle and (b) yaw rate before saddle-node point at $\nu=72 \mathrm{~km} / \mathrm{h}, \delta_{\mathrm{f}}=-9.09^{\circ}$. Vehicle states (c) slip angle and (d) yaw rate after saddle-node point at $\nu=72 \mathrm{~km} / \mathrm{h}, \delta_{\mathrm{f}}=-9.14^{\circ}$.

where $O(2)$ denotes the remaining second-order term and other high-order terms

$$
g \triangleq\left[\begin{array}{l}
g_{1} \\
g_{2}
\end{array}\right]=\left[\begin{array}{c}
\frac{1}{m \nu} \frac{\partial}{\partial \delta_{\mathrm{f}}}\left(F_{y \mathrm{f}}+F_{y \mathrm{r}}\right)\left(x^{0}, \delta_{\mathrm{f}}^{0}\right) \\
\frac{\cos \beta^{0}}{I_{z}} \frac{\partial}{\partial \delta_{\mathrm{f}}}\left(L_{\mathrm{f}} F_{y \mathrm{f}}-L_{\mathrm{r}} F_{y \mathrm{r}}\right)\left(x^{0}, \delta_{\mathrm{f}}^{0}\right)
\end{array}\right]
$$

and

$$
Q(\tilde{x}, \tilde{x})=\left[\begin{array}{l}
q_{11} x_{1}^{2}+q_{12} x_{1} x_{2}+q_{13} x_{2}^{2} \\
q_{21} x_{1}^{2}+q_{22} x_{1} x_{2}+q_{23} x_{2}^{2}
\end{array}\right] .
$$

The Jacobian matrix $A$ is the same as the one in (10) but with $a_{1} a_{4}=a_{2} a_{3}$, and the values of $q_{i j}$ are given in Appendix A. From Observation 1, matrix $A$ has one zero eigenvalue and a stable eigenvalue for a general vehicle. In the following, we assume $a_{1}<0$ and $a_{4}<0$ without loss of generality.

Define $l$ and $r$ as the left and right eigenvectors corresponding to the zero eigenvalues of $A$ with $l r=1$. We then have

$$
l=\left[\frac{a_{4}}{a_{1}+a_{4}}-\frac{a_{2}}{a_{1}+a_{4}}\right]
$$

and

$$
r=\left[\begin{array}{c}
1 \\
-\frac{a_{1}}{a_{2}}
\end{array}\right]^{\mathrm{T}}
$$

It is clear from the relation of $a_{1} a_{4}=a_{2} a_{3}$ that we can rewrite $r$ as

$$
r=\left[\begin{array}{c}
1 \\
-\frac{a_{3}}{a_{4}}
\end{array}\right]^{\mathrm{T}}
$$

By applying Lemma 2 to system (16), we have

$$
l \cdot g=\frac{1}{a_{1}+a_{4}}\left(a_{4} g_{1}-a_{2} g_{2}\right)
$$

and

$$
\begin{aligned}
l Q_{0}(r, r)= & {\left[\frac{a_{4}}{a_{1}+a_{4}}-\frac{a_{2}}{a_{1}+a_{4}}\right] } \\
& \cdot\left[\begin{array}{l}
\left.q_{11}+q_{12}\left(-\frac{a_{3}}{a_{4}}\right)+q_{13}\left(-\frac{a_{3}}{a_{4}}\right)^{2}\right] \\
\left.q_{21}+q_{22}\left(-\frac{a_{1}}{a_{2}}\right)+q_{23}\left(-\frac{a_{1}}{a_{2}}\right)^{2}\right]
\end{array}\right. \\
= & \frac{1}{a_{1}+a_{4}}\left(a_{4} q_{11}-a_{3} q_{12}+\frac{a_{3}^{2}}{a_{4}} q_{13}\right. \\
& \left.\quad-a_{2} q_{21}+a_{1} q_{22}-\frac{a_{1}^{2}}{a_{2}} q_{23}\right) .
\end{aligned}
$$

The next result follows readily from Lemma 2. 


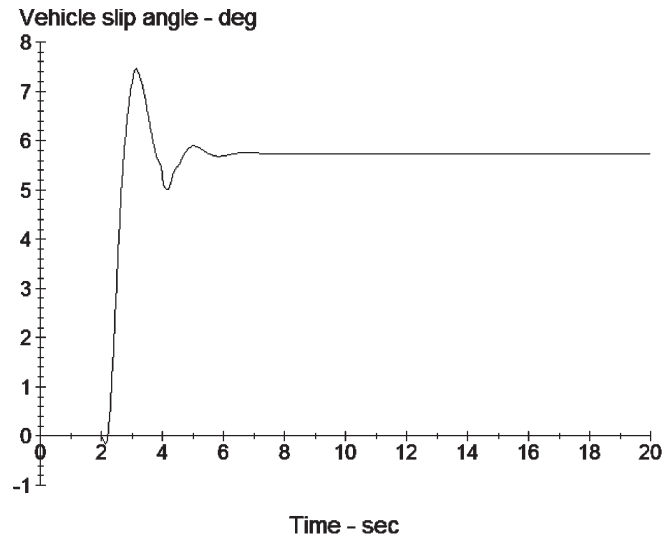

(a)

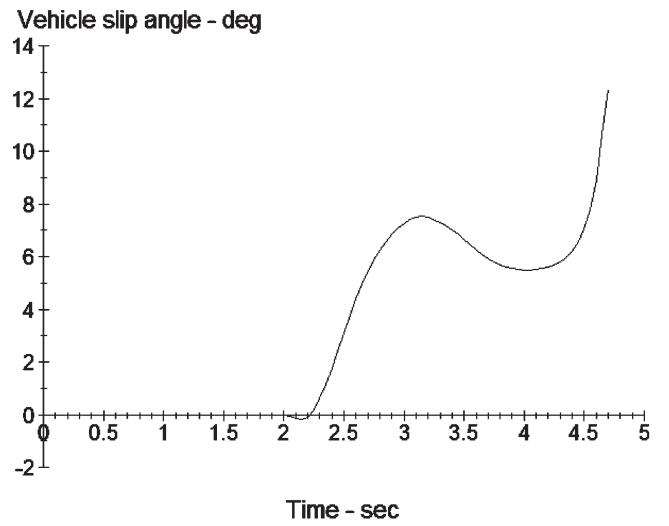

(c)

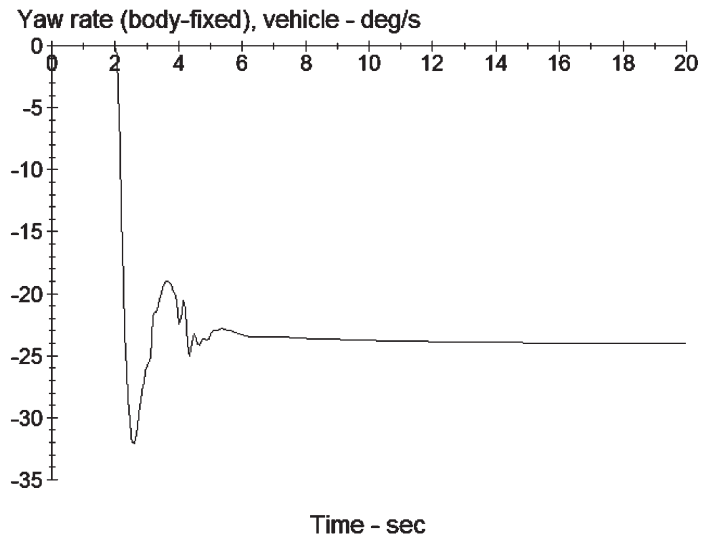

(b)

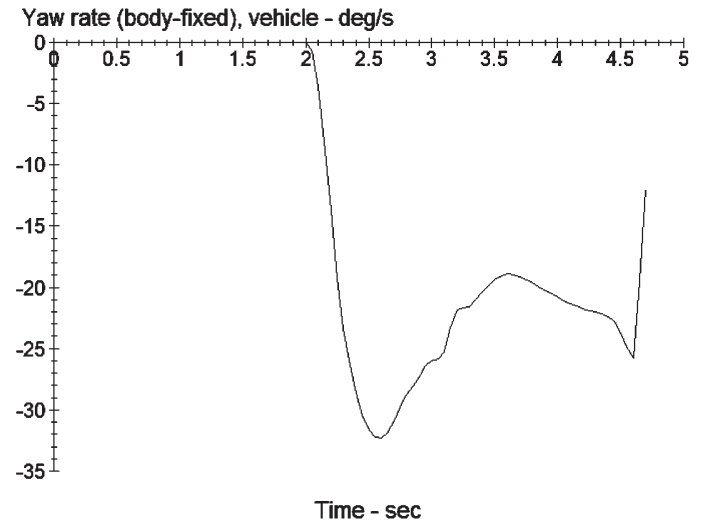

(d)

Fig. 9. Vehicle states (a) slip angle and (b) yaw rate before saddle-node point at $\nu=108 \mathrm{~km} / \mathrm{h}, \delta_{\mathrm{f}}=-4.89^{\circ}$. Vehicle states (c) slip angle and (d) yaw rate after saddle-node point at $\nu=108 \mathrm{~km} / \mathrm{h}, \delta_{\mathrm{f}}=-4.93^{\circ}$.

Theorem 1: The system (4), (5) will undergo saddle-node bifurcation for the equilibrium point $x^{0}$ if the following conditions hold:

1) $a_{1} a_{4}=a_{2} a_{3}$ with $a_{1}<0$ and $a_{4}<0$;

2) $a_{4} g_{1} \neq a_{2} g_{2}$;

3) $a_{4} q_{11}-a_{3} q_{12}+\left(a_{3}^{2} / a_{4}\right) q_{13}-a_{2} q_{21}+a_{1} q_{22}-$ $\left(a_{1}^{2} / a_{2}\right) q_{23} \neq 0$.

\section{Application to the Vehicle Dynamics With Magic Formula Type Cornering Force}

There are many models for cornering force. In this paper, we adopt the well-known "Magic formula" mathematical model (e.g., [7]) for the nonlinear cornering forces $F_{y \mathrm{f}}$ and $F_{y \mathrm{r}}$ as

$F_{y \mathrm{f}}=D_{\mathrm{f}} \sin \left[C_{\mathrm{f}} \tan ^{-1}\left\{B_{\mathrm{f}}\left(1-E_{\mathrm{f}}\right) \alpha_{\mathrm{f}}+E_{\mathrm{f}} \tan ^{-1}\left(B_{\mathrm{f}} \alpha_{\mathrm{f}}\right)\right\}\right]$

$F_{y \mathrm{r}}=D_{\mathrm{r}} \sin \left[C_{\mathrm{r}} \tan ^{-1}\left\{B_{\mathrm{r}}\left(1-E_{\mathrm{r}}\right) \alpha_{\mathrm{r}}+E_{\mathrm{r}} \tan ^{-1}\left(B_{\mathrm{r}} \alpha_{\mathrm{r}}\right)\right\}\right]$

where

$$
\alpha_{\mathrm{f}}=\beta+\tan ^{-1}\left(\frac{L_{\mathrm{f}}}{\nu} r \cdot \cos \beta\right)-\delta_{\mathrm{f}}
$$

and

$$
\alpha_{\mathrm{r}}=\beta-\tan ^{-1}\left(\frac{L_{\mathrm{r}}}{\nu} r \cdot \cos \beta\right) .
$$

Here, $\alpha_{\mathrm{f}}$ and $\alpha_{\mathrm{r}}$ denote the slip angle for the front and rear tires, respectively. The approximation relationship between tire slip angles and cornering forces are depicted in Fig. 2.

It is clear from $(21)$ that $x=(\beta, r)^{\mathrm{T}}=(0,0)^{\mathrm{T}}$ will make $F_{y \mathrm{f}}=F_{y \mathrm{r}}=0$ when $\delta_{\mathrm{f}}^{0}=0$. Thus, $x^{0}=(0,0)^{\mathrm{T}}$ is an equilibrium point for system (4), (5) for $\delta_{\mathrm{f}}^{0}=0$. This agrees with the natural behavior of vehicle dynamics. In the following, we first consider to solve the approximation of equilibrium solution about $x^{0}=(0,0)^{\mathrm{T}}$.

It is known that $\theta \approx \tan \theta, \sin \theta \approx \theta$, and $\cos \theta \approx 1$ for $\theta \approx 0$. By setting $\dot{x}=0$ to solve for the equilibrium solution $x^{0}=\left(\beta^{0}, r^{0}\right)^{\mathrm{T}}$ of system (4), (5) near $x^{0}=(0,0)^{\mathrm{T}}$, we have, from (21), that

$$
D_{\mathrm{f}} C_{\mathrm{f}} B_{\mathrm{f}}\left(\beta^{0}+\frac{L_{\mathrm{f}}}{\nu} r^{0}-\delta_{\mathrm{f}}^{0}\right)+D_{\mathrm{r}} C_{\mathrm{r}} B_{\mathrm{r}}\left(\beta^{0}-\frac{L_{\mathrm{r}}}{\nu} r^{0}\right)=m \nu r^{0}
$$

$$
L_{\mathrm{f}} D_{\mathrm{f}} C_{\mathrm{f}} B_{\mathrm{f}}\left(\beta^{0}+\frac{L_{\mathrm{f}}}{\nu} r^{0}-\delta_{\mathrm{f}}^{0}\right)-L_{\mathrm{r}} D_{\mathrm{r}} C_{\mathrm{r}} B_{\mathrm{r}}\left(\beta^{0}-\frac{L_{\mathrm{r}}}{\nu} r^{0}\right)=0 .
$$




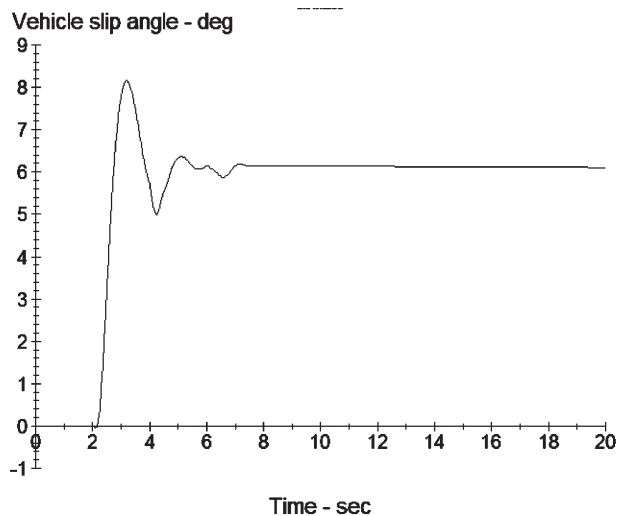

(a)

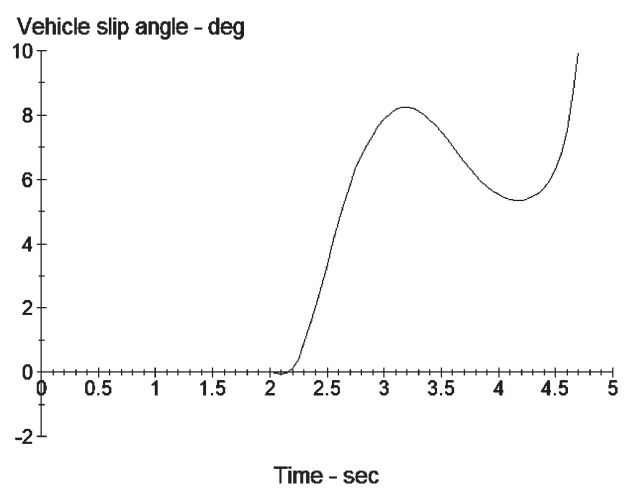

(c)

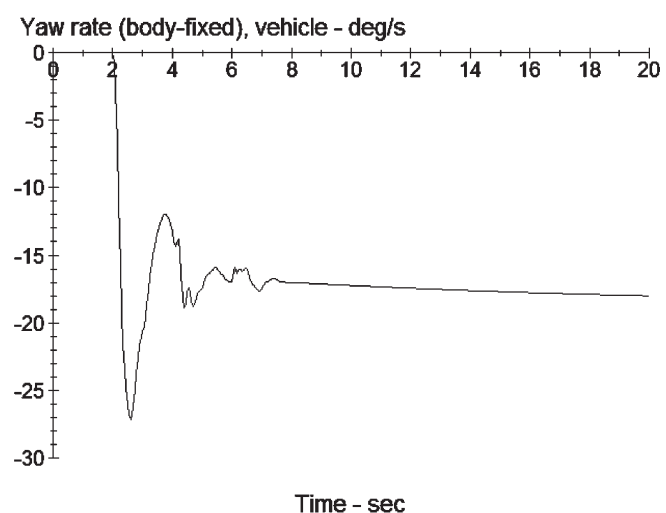

(b)

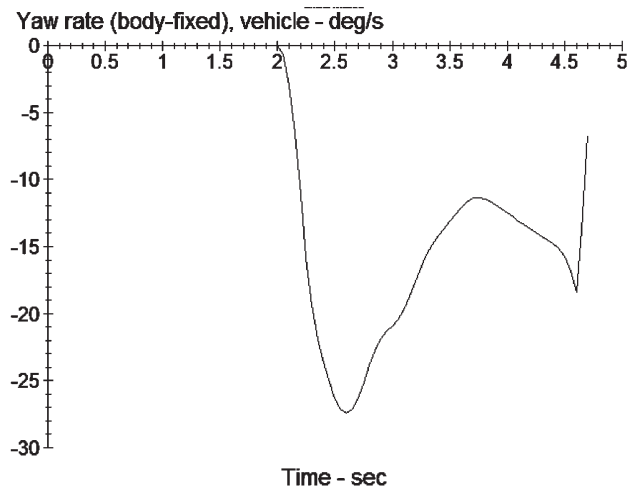

(d)

Fig. 10. Vehicle states (a) slip angle and (b) yaw rate before saddle-node point at $\nu=144 \mathrm{~km} / \mathrm{h}, \delta_{\mathrm{f}}=-2.91^{\circ}$. Vehicle states (c) slip angle and (d) yaw rate after saddle-node point at $\nu=144 \mathrm{~km} / \mathrm{h}, \delta_{\mathrm{f}}=-2.94^{\circ}$.

By suitable manipulation, we can then rewrite (23) and (24) as

$$
\left(L_{\mathrm{f}}+L_{\mathrm{r}}\right) D_{\mathrm{f}} C_{\mathrm{f}} B_{\mathrm{f}}\left(\beta^{0}+\frac{L_{\mathrm{f}}}{\nu} r^{0}-\delta_{\mathrm{f}}^{0}\right)=L_{\mathrm{r}} m \nu r^{0}
$$

and

$$
\left(L_{\mathrm{f}}+L_{\mathrm{r}}\right) D_{\mathrm{r}} C_{\mathrm{r}} B_{\mathrm{r}}\left(\beta^{0}-\frac{L_{\mathrm{r}}}{\nu} r^{0}\right)=L_{\mathrm{f}} m \nu r^{0} .
$$

The following observation can be made from (25) and (26).

Observation 2: In general, the values of constants $B_{\mathrm{f}}, B_{\mathrm{r}}$, $C_{\mathrm{f}}$, and $C_{\mathrm{r}}$ are positive, while those of $D_{\mathrm{f}}$ and $D_{\mathrm{r}}$ are negative. Example values of these variables will be given in Section IV. Thus, it is not difficult to find from (25) and (26) that the values of $\beta^{0}$ and $r^{0}$ will have the same sign if

$$
\nu<\nu_{\mathrm{ss}}=\sqrt{\frac{-L_{\mathrm{r}}\left(L_{\mathrm{f}}+L_{\mathrm{r}}\right) D_{\mathrm{r}} C_{\mathrm{r}} B_{\mathrm{r}}}{L_{\mathrm{f}} m}}
$$

and the sign of the sideslip angle $\beta^{0}$ will be opposite from that of the yaw rate $r^{0}$ for large axial velocity $\nu$.

Next, we consider the stability of system (4), (5) at the equilibrium point $x^{0}=(0,0)^{\mathrm{T}}$ by using Lemma 1 . According to the Magic formula as given in (21) and (22), the values of $a_{i}$ for the Jacobian matrix $A$ at the equilibrium point $x^{0}=\left(\beta^{0}, \gamma^{0}\right)^{\mathrm{T}}$ are calculated as those given in Appendix B. For the case of $x^{0}=(0,0)^{\mathrm{T}}$, we have from Observation 2

$$
\begin{aligned}
& a_{1}=\frac{1}{m \nu}\left(D_{\mathrm{f}} C_{\mathrm{f}} B_{\mathrm{f}}+D_{\mathrm{r}} C_{\mathrm{r}} B_{\mathrm{r}}\right)<0 \\
& a_{2}=\frac{1}{m \nu^{2}}\left(L_{\mathrm{f}} D_{\mathrm{f}} C_{\mathrm{f}} B_{\mathrm{f}}-L_{\mathrm{r}} D_{\mathrm{r}} C_{\mathrm{r}} B_{\mathrm{r}}\right)-1<0 \\
& a_{3}=\frac{1}{I_{z}}\left(L_{\mathrm{f}} D_{\mathrm{f}} C_{\mathrm{f}} B_{\mathrm{f}}-L_{\mathrm{r}} D_{\mathrm{r}} C_{\mathrm{r}} B_{\mathrm{r}}\right)>0
\end{aligned}
$$

and

$$
a_{4}=\frac{1}{I_{z} \nu}\left(L_{\mathrm{f}}^{2} D_{\mathrm{f}} C_{\mathrm{f}} B_{\mathrm{f}}+L_{\mathrm{r}}^{2} D_{\mathrm{r}} C_{\mathrm{r}} B_{\mathrm{r}}\right)<0
$$

It is clear from (28)-(31) that $a_{1}<0, a_{2}<0, a_{3}>0$, and $a_{4}<0$ for practical values of $B_{i}, C_{i}, D_{i}(i=f, r)$. One example is given in Section IV. These agree with the discussions in Observation 1. The next stability result follows readily from Lemma 1.

Corollary 1: The equilibrium point $x^{0}=(0,0)^{\mathrm{T}}$ for $\delta_{\mathrm{f}}^{0}$ is asymptotically stable.

Since the equilibrium point $x^{0}=(0,0)^{\mathrm{T}}$ is asymptotically stable, the bifurcation phenomena will not emerge from it. According to (21) and (22), $g_{i}$ and $q_{i j}$ are also calculated and given in Appendix B. Based on those values of $a_{i}, g_{i}$, and $q_{i j}$ given in Appendix B, it is not difficult to apply Theorem 1 to the study of existence conditions of saddle-node bifurcation for system (4), (5). Numerical examples are given in Section IV for finding such a possibility. 


\section{Numerical Simulations}

In this section, we present the numerical study of lateral vehicle dynamics as given in (4) and (5). The selected values of system parameters given in (21) and (22) are the same as those of [6]. Details are given in Tables I and II. In order to verify the study of this paper, the system parameters for low-friction roads are chosen such that the vehicle has a propensity to spin. This could conform the driving condition to that of traveling down hill at constant velocity with equivalent braking effect at zero throttle being applied.

As discussed in Section III, the applied front wheel angle $\delta_{\mathrm{f}}$ is treated as a bifurcation parameter. Computer code AUTO [20] is employed to do the numerical analysis since it can numerically calculate the system equilibrium branches by the property of continuity, the eigenvalues of the Jacobian matrix at each equilibrium point, and then determine the corresponding system stability. It is known (e.g., [7], [13], and [14]) that a nonlinear system will have the property of stability exchange before and after the stationary bifurcation point and possess a new system equilibrium branch with respect to the variation of bifurcation parameter. This gives a guide to determine the occurrence of stationary bifurcation and used in AUTO software.

The bifurcation diagram of system (4), (5) with respect to the variation of $\delta_{\mathrm{f}}$ is shown in Fig. 3. Note that in Fig. 3, the solid line denotes the stable equilibrium point while the dashed line is for the unstable equilibrium point. As depicted in Fig. 3(a) and (b), the system equilibrium near the origin are all asymptotically stable for different driving speeds $\nu$. This agrees with the analytical result given in Corollary 1 . As discussed in Observation 2, the equilibrium values of $\beta$ and $\gamma$ will have the same sign if the value of velocity $\nu$ is less than $\nu_{\mathrm{ss}} \approx 9.5 \mathrm{~m} / \mathrm{s}$. Figs. 3 and 4 demonstrate such results. Saddle-node bifurcation emerging from the equilibrium solution of system (4), (5) is also observed in Fig. 3. The locations of the bifurcation points for different driving speeds, $\nu$ are obtained by using AUTO and given in Table III. It is clear from Fig. 3 that the system equilibrium changes stability at the saddle-node bifurcation point and the magnitude of $\delta_{\mathrm{f}}$ corresponding to the saddle-node bifurcation becomes smaller as the velocity $\nu$ increases.

Note that the equilibrium points within the range of $\delta_{\mathrm{f}}$ between the two saddle nodes are stable. It should be pointed out that the equilibrium of sideslip angle is divergent while the yaw rate is convergent for increasing $\delta_{\mathrm{f}}$ in the bifurcation diagram. Figs. 5 and 6, respectively, show the time responses for two initial conditions with $\nu=20 \mathrm{~m} / \mathrm{s}$ for different values of $\delta_{\mathrm{f}}$. It can be seen that the vehicle is stable and ends at the value corresponding to the equilibrium point $x^{0}$ within the range $\left|\delta_{\mathrm{f}}\right|<0.0158$ rad. It is observed in Figs. 5 and 6 that the system becomes unstable when $\left|\delta_{\mathrm{f}}\right|>0.0158 \mathrm{rad}$. This is attributed by the existence of saddle-node bifurcation and its corresponding stability property (e.g., [7], [11], and [12]).

The bifurcation analysis obtained in Section III is employed to verify the numerical results. As given in Table IV, the saddlenode bifurcation point for $\nu=20 \mathrm{~m} / \mathrm{s}$ does satisfy the existence conditions of Theorem 1. Thus, the analytical results presented in Section III will be very helpful in finding possible bifurcation scenarios and system stability. In addition, the location of

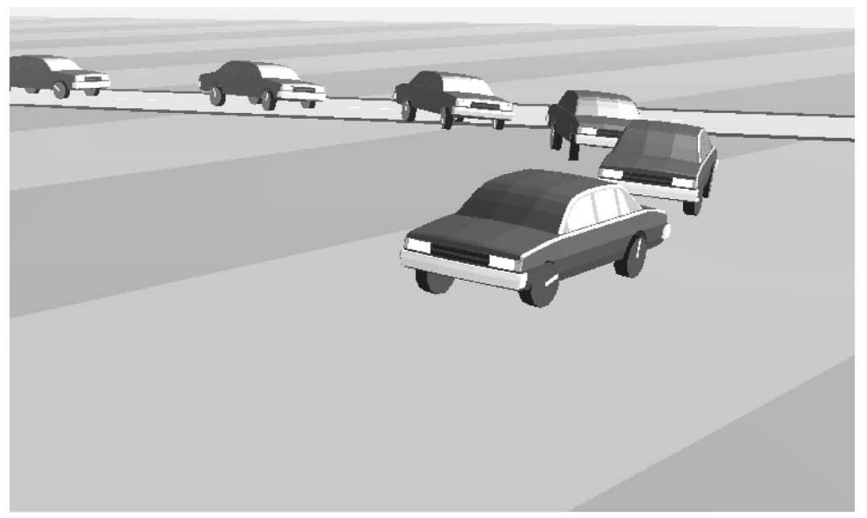

(a)

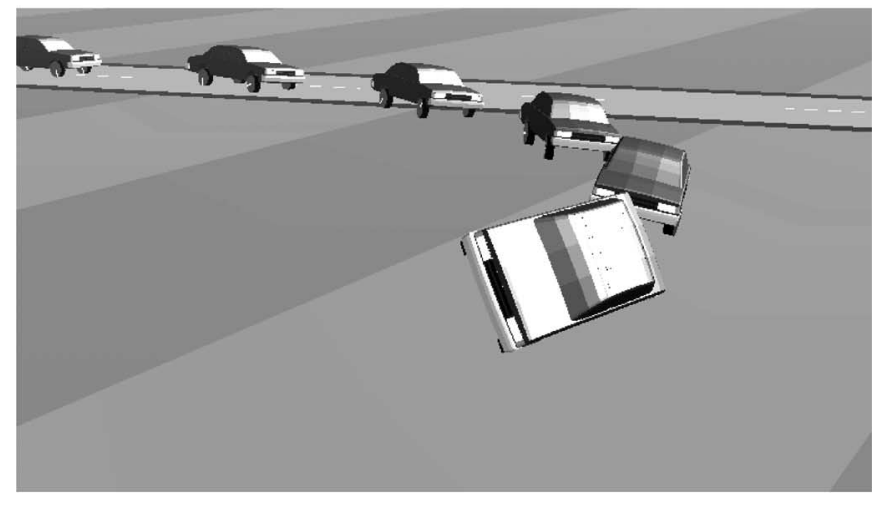

(b)

Fig. 11. Vehicle animation at $\nu=72 \mathrm{~km} / \mathrm{h}$ in CarSim (a) before saddlenode bifurcation point $\left(\delta_{\mathrm{f}}=-9.09^{\circ}\right)$. (b) After saddle-node bifurcation point $\left(\delta_{\mathrm{f}}=-9.14^{\circ}\right)$.

saddle-node bifurcation for values of $\delta_{\mathrm{f}}$ with respect to the variation in velocity $\nu$ is also given in Fig. 7 .

Similar phenomena can be found by using the famous vehicle simulation software CarSim. An example of using the code CarSim to find the occurrence of saddle-node bifurcation is depicted in Figs. 8-10. It is observed from Figs. 8-10 that both slip angle and yaw rate will reach stable values before the saddle-node bifurcation point but become unstable after the bifurcation point for three different values of velocity. Note that all the simulations presented in Figs. 8-10 are obtained by sending the steering command at time $t=2 \mathrm{~s}$. Computer animations of the vehicle's behavior before and after the bifurcation point are also obtained using CarSim and depicted in Fig. 11 to demonstrate the existence how the existence of saddle-node bifurcation might affect the vehicle's stability. The location of saddle-node bifurcation in two-parameter space is also obtained as given in Fig. 12.

\section{CONCLUSion}

In this paper, we focused on the study of stability and nonlinear behavior of vehicle lateral dynamics. This is achieved by applying the Routh-Hurwitz stability criterion and bifurcation theory to the second-order model of lateral vehicle dynamics. Without assuming small angles, the stability condition at a given constant velocity for steering systems is also derived, which depends heavily on the cornering force characteristics of 


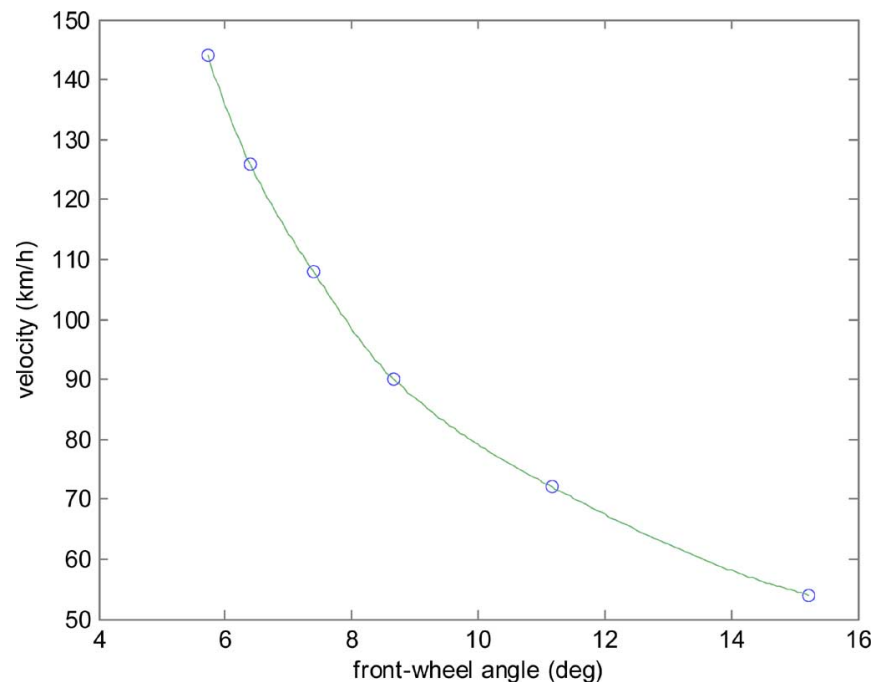

Fig. 12. Saddle-node bifurcation point in $\left(\nu-\left|\delta_{\mathrm{f}}\right|\right)$ plane for CarSim vehicle model.

the rear tires. Saddle-node bifurcation phenomenon is observed from the equilibrium solution of the nonlinear model, while it never appears in the linear bicycle model (e.g., [3]-[5]). It is found by this paper that the velocity plays a very important role in determining the location of bifurcation points, which is not discussed in the existing results (e.g., [6]). In fact, the value of the applied front wheel angle for the bifurcation points is found to be smaller as the velocity increases. This may provide a partial reason as to why the vehicle steering is hard to control and easily becomes unstable under high speeds.

\section{APPENDIX A}

The values of $q_{i j}$ are given as follows:

$$
q_{11}=\frac{1}{m \nu}\left(\frac{\partial^{2}}{\partial \beta^{2}}\left(F_{y \mathrm{f}}+F_{y \mathrm{r}}\right)\left(\beta^{0}, r^{0}, \delta_{\mathrm{f}}^{0}\right)\right)
$$

$$
\begin{aligned}
& q_{12}=\frac{1}{m \nu}\left(\frac{\partial^{2}}{\partial \beta \partial r}\left(F_{y \mathrm{f}}+F_{y \mathrm{r}}\right)\left(\beta^{0}, r^{0}, \delta_{\mathrm{f}}^{0}\right)\right) \\
& q_{13}=\frac{1}{m \nu}\left(\frac{\partial^{2}}{\partial r^{2}}\left(F_{y \mathrm{f}}+F_{y \mathrm{r}}\right)\left(\beta^{0}, r^{0}, \delta_{\mathrm{f}}^{0}\right)\right) \\
& q_{21}=\frac{1}{I_{z}}\left(\left(\frac{\partial^{2}}{\partial \beta^{2}} L_{\mathrm{f}} F_{y \mathrm{f}}\left(\beta^{0}, r^{0}, \delta_{\mathrm{f}}^{0}\right)\right.\right. \\
& \left.-\frac{\partial^{2}}{\partial \beta^{2}} L_{\mathrm{r}} F_{y \mathrm{r}}\left(\beta^{0}, r^{0}, \delta_{\mathrm{f}}^{0}\right)\right) \cos \beta^{0} \\
& -2 \cdot\left(\frac{\partial}{\partial \beta} L_{\mathrm{f}} F_{y \mathrm{f}}\left(\beta^{0}, r^{0}, \delta_{\mathrm{f}}^{0}\right)\right. \\
& \left.\left.-\frac{\partial}{\partial \beta} L_{\mathrm{r}} F_{y \mathrm{r}}\left(\beta^{0}, r^{0}, \delta_{\mathrm{f}}^{0}\right)\right) \sin \beta^{0}\right) \\
& q_{22}=\frac{1}{I_{z}}\left(\left(\frac{\partial^{2}}{\partial \beta \partial r} L_{\mathrm{f}} F_{y \mathrm{f}}\left(\beta^{0}, r^{0}, \delta_{\mathrm{f}}^{0}\right)\right.\right. \\
& \left.-\frac{\partial^{2}}{\partial \beta \partial r} L_{\mathrm{r}} F_{y \mathrm{r}}\left(\beta^{0}, r^{0}, \delta_{\mathrm{f}}^{0}\right)\right) \cos \beta^{0} \\
& -\left(\frac{\partial}{\partial r} L_{\mathrm{f}} F_{y \mathrm{f}}\left(\beta^{0}, r^{0}, \delta_{\mathrm{f}}^{0}\right)\right. \\
& \left.\left.-\frac{\partial}{\partial r} L_{\mathrm{r}} F_{y \mathrm{r}}\left(\beta^{0}, r^{0}, \delta_{\mathrm{f}}^{0}\right)\right) \sin \beta^{0}\right) \\
& q_{23}=\frac{1}{I_{z}}\left(\frac{\partial^{2}}{\partial r^{2}} L_{\mathrm{f}} F_{y \mathrm{f}}\left(\beta^{0}, r^{0}, \delta_{\mathrm{f}}^{0}\right)\right. \\
& \left.-\frac{\partial^{2}}{\partial r^{2}} L_{\mathrm{r}} F_{y \mathrm{r}}\left(\beta^{0}, r^{0}, \delta_{\mathrm{f}}^{0}\right)\right) \cos \beta^{0} .
\end{aligned}
$$

\section{APPENDIX B}

The values of $a_{i}$ and $g_{i}$ are shown at the bottom of the page and are continued on the next page, where $\nu, m, I_{z}, L_{j}, B_{j}, C_{j}$, $D_{j}, E_{j}(j=f, r)$ are as defined in previous sections.

$$
\begin{aligned}
a_{1}=\frac{1}{m \nu}( & D_{\mathrm{f}} \cos \left[C_{\mathrm{f}} \tan ^{-1}\left\{B_{\mathrm{f}}\left(1-E_{\mathrm{f}}\right) \alpha_{\mathrm{f}}\left(\beta^{0}, r^{0}, \delta_{\mathrm{f}}^{0}\right)+E_{\mathrm{f}} \tan ^{-1}\left(B_{\mathrm{f}} \alpha_{\mathrm{f}}\left(\beta^{0}, r^{0}, \delta_{\mathrm{f}}^{0}\right)\right)\right\}\right] \\
& \cdot\left[\frac{C_{\mathrm{f}}}{1+\left(B_{\mathrm{f}}\left(1-E_{\mathrm{f}}\right) \alpha_{\mathrm{f}}\left(\beta^{0}, r^{0}, \delta_{\mathrm{f}}^{0}\right)+E_{\mathrm{f}} \tan ^{-1}\left(B_{\mathrm{f}} \alpha_{\mathrm{f}}\left(\beta^{0}, r^{0}, \delta_{\mathrm{f}}^{0}\right)\right)\right)^{2}}\right] \\
& \cdot\left[B_{\mathrm{f}}\left(1-E_{\mathrm{f}}\right)+\frac{E_{\mathrm{f}} B_{\mathrm{f}}}{1+\left(B_{\mathrm{f}} \alpha_{\mathrm{f}}\left(\beta^{0}, r^{0}, \delta_{\mathrm{f}}^{0}\right)\right)^{2}}\right] \cdot\left[1-\frac{\frac{L_{\mathrm{f}}}{\nu} r^{0} \sin \beta^{0}}{1+\left(\frac{L_{\mathrm{f}}}{\nu} r^{0} \cos \beta^{0}\right)^{2}}\right] \\
+ & D_{\mathrm{r}} \cos \left[C_{\mathrm{r}} \tan ^{-1}\left\{B_{\mathrm{r}}\left(1-E_{\mathrm{r}}\right) \alpha_{\mathrm{r}}\left(\beta^{0}, r^{0}\right)+E_{\mathrm{r}} \tan ^{-1}\left(B_{\mathrm{r}} \alpha_{\mathrm{r}}\left(\beta^{0}, r^{0}\right)\right)\right\}\right] \\
& \cdot\left[\frac{C_{\mathrm{r}}}{1+\left(B_{\mathrm{r}}\left(1-E_{\mathrm{r}}\right) \alpha_{\mathrm{r}}\left(\beta^{0}, r^{0}\right)+E_{\mathrm{r}} \tan ^{-1}\left(B_{\mathrm{r}} \alpha_{\mathrm{r}}\left(\beta^{0}, r^{0}\right)\right)\right)^{2}}\right] \\
& \left.\cdot\left[B_{\mathrm{r}}\left(1-E_{\mathrm{r}}\right)+\frac{E_{\mathrm{r}} B_{\mathrm{r}}}{1+\left(B_{\mathrm{r}} \alpha_{\mathrm{r}}\left(\beta^{0}, r^{0}\right)\right)^{2}}\right] \cdot\left[1+\frac{\frac{L_{\mathrm{r}}}{\nu} r^{0} \sin \beta^{0}}{1+\left(\frac{L_{\mathrm{r}}}{\nu} r^{0} \cos \beta^{0}\right)^{2}}\right]\right)
\end{aligned}
$$




$$
\begin{aligned}
& a_{2}=\frac{1}{m \nu}\left(D_{\mathrm{f}} \cos \left[C_{\mathrm{f}} \tan ^{-1}\left\{B_{\mathrm{f}}\left(1-E_{\mathrm{f}}\right) \alpha_{\mathrm{f}}\left(\beta^{0}, r^{0}, \delta_{\mathrm{f}}^{0}\right)+E_{\mathrm{f}} \tan ^{-1}\left(B_{\mathrm{f}} \alpha_{\mathrm{f}}\left(\beta^{0}, r^{0}, \delta_{\mathrm{f}}^{0}\right)\right)\right\}\right]\right. \\
& \cdot\left[\frac{C_{\mathrm{f}}}{1+\left(B_{\mathrm{f}}\left(1-E_{\mathrm{f}}\right) \alpha_{\mathrm{f}}\left(\beta^{0}, r^{0}, \delta_{\mathrm{f}}^{0}\right)+E_{\mathrm{f}} \tan ^{-1}\left(B_{\mathrm{f}} \alpha_{\mathrm{f}}\left(\beta^{0}, r^{0}, \delta_{\mathrm{f}}^{0}\right)\right)\right)^{2}}\right] \\
& \cdot\left[B_{\mathrm{f}}\left(1-E_{\mathrm{f}}\right)+\frac{E_{\mathrm{f}} B_{\mathrm{f}}}{1+\left(B_{\mathrm{f}} \alpha_{\mathrm{f}}\left(\beta^{0}, r^{0}, \delta_{\mathrm{f}}^{0}\right)\right)^{2}}\right] \cdot\left[\frac{\frac{L_{\mathrm{f}}}{\nu} \cos \beta^{0}}{1+\left(\frac{L_{\mathrm{f}}}{\nu} r^{0} \cos \beta^{0}\right)^{2}}\right] \\
& +D_{\mathrm{r}} \cos \left[C_{\mathrm{r}} \tan ^{-1}\left\{B_{\mathrm{r}}\left(1-E_{\mathrm{r}}\right) \alpha_{\mathrm{r}}\left(\beta^{0}, r^{0}\right)+E_{\mathrm{r}} \tan ^{-1}\left(B_{\mathrm{r}} \alpha_{\mathrm{r}}\left(\beta^{0}, r^{0}\right)\right)\right\}\right] \\
& \cdot\left[\frac{C_{\mathrm{r}}}{1+\left(B_{\mathrm{r}}\left(1-E_{\mathrm{r}}\right) \alpha_{\mathrm{r}}\left(\beta^{0}, r^{0}\right)+E_{\mathrm{r}} \tan ^{-1}\left(B_{\mathrm{r}} \alpha_{\mathrm{r}}\left(\beta^{0}, r^{0}\right)\right)\right)^{2}}\right] \\
& \left.\cdot\left[B_{\mathrm{r}}\left(1-E_{\mathrm{r}}\right)+\frac{E_{\mathrm{r}} B_{\mathrm{r}}}{1+\left(B_{\mathrm{r}} \alpha_{\mathrm{r}}\left(\beta^{0}, r^{0}\right)\right)^{2}}\right] \cdot\left[-\frac{\frac{L_{\mathrm{r}}}{\nu} \cos \beta^{0}}{1+\left(\frac{L_{\mathrm{r}}}{\nu} r^{0} \cos \beta^{0}\right)^{2}}\right]\right)-1 \\
& a_{3}=\frac{\cos \beta}{I_{z}}\left(L_{\mathrm{f}} D_{\mathrm{f}} \cos \left[C_{\mathrm{f}} \tan ^{-1}\left\{B_{\mathrm{f}}\left(1-E_{\mathrm{f}}\right) \alpha_{\mathrm{f}}\left(\beta^{0}, r^{0}, \delta_{\mathrm{f}}^{0}\right)+E_{\mathrm{f}} \tan ^{-1}\left(B_{\mathrm{f}} \alpha_{\mathrm{f}}\left(\beta^{0}, r^{0}, \delta_{\mathrm{f}}^{0}\right)\right)\right\}\right]\right. \\
& \cdot\left[\frac{C_{\mathrm{f}}}{1+\left(B_{\mathrm{f}}\left(1-E_{\mathrm{f}}\right) \alpha_{\mathrm{f}}\left(\beta^{0}, r^{0}, \delta_{\mathrm{f}}^{0}\right)+E_{\mathrm{f}} \tan ^{-1}\left(B_{\mathrm{f}} \alpha_{\mathrm{f}}\left(\beta^{0}, r^{0}, \delta_{\mathrm{f}}^{0}\right)\right)\right)^{2}}\right] \\
& \cdot\left[B_{\mathrm{f}}\left(1-E_{\mathrm{f}}\right)+\frac{E_{\mathrm{f}} B_{\mathrm{f}}}{1+\left(B_{\mathrm{f}} \alpha_{\mathrm{f}}\left(\beta^{0}, r^{0}, \delta_{\mathrm{f}}^{0}\right)\right)^{2}}\right] \cdot\left[1-\frac{\frac{L_{\mathrm{f}}}{\nu} r^{0} \sin \beta^{0}}{1+\left(\frac{L_{\mathrm{f}}}{\nu} r^{0} \cos \beta^{0}\right)^{2}}\right] \\
& -L_{\mathrm{r}} D_{\mathrm{r}} \cos \left[C_{\mathrm{r}} \tan ^{-1}\left\{B_{\mathrm{r}}\left(1-E_{\mathrm{r}}\right) \alpha_{\mathrm{r}}\left(\beta^{0}, r^{0}\right)+E_{\mathrm{r}} \tan ^{-1}\left(B_{\mathrm{r}} \alpha_{\mathrm{r}}\left(\beta^{0}, r^{0}\right)\right)\right\}\right] \\
& \cdot\left[\frac{C_{\mathrm{r}}}{1+\left(B_{\mathrm{r}}\left(1-E_{\mathrm{r}}\right) \alpha_{\mathrm{r}}\left(\beta^{0}, r^{0}\right)+E_{\mathrm{r}} \tan ^{-1}\left(B_{\mathrm{r}} \alpha_{\mathrm{r}}\left(\beta^{0}, r^{0}\right)\right)\right)^{2}}\right] \\
& \left.\cdot\left[B_{\mathrm{r}}\left(1-E_{\mathrm{r}}\right)+\frac{E_{\mathrm{r}} B_{\mathrm{r}}}{1+\left(B_{\mathrm{r}} \alpha_{\mathrm{r}}\left(\beta^{0}, r^{0}\right)\right)^{2}}\right] \cdot\left[1+\frac{\frac{L_{\mathrm{r}}}{\nu} r^{0} \sin \beta^{0}}{1+\left(\frac{L_{\mathrm{r}}}{\nu} r^{0} \cos \beta^{0}\right)^{2}}\right]\right) \\
& a_{4}=\frac{\cos \beta}{I_{z}}\left(L_{\mathrm{f}} D_{\mathrm{f}} \cos \left[C_{\mathrm{f}} \tan ^{-1}\left\{B_{\mathrm{f}}\left(1-E_{\mathrm{f}}\right) \alpha_{\mathrm{f}}\left(\beta^{0}, r^{0}, \delta_{\mathrm{f}}^{0}\right)+E_{\mathrm{f}} \tan ^{-1}\left(B_{\mathrm{f}} \alpha_{\mathrm{f}}\left(\beta^{0}, r^{0}, \delta_{\mathrm{f}}^{0}\right)\right)\right\}\right]\right. \\
& \cdot\left[\frac{C_{\mathrm{f}}}{1+\left(B_{\mathrm{f}}\left(1-E_{\mathrm{f}}\right) \alpha_{\mathrm{f}}\left(\beta^{0}, r^{0}, \delta_{\mathrm{f}}^{0}\right)+E_{\mathrm{f}} \tan ^{-1}\left(B_{\mathrm{f}} \alpha_{\mathrm{f}}\left(\beta^{0}, r^{0}, \delta_{\mathrm{f}}^{0}\right)\right)\right)^{2}}\right] \\
& \cdot\left[B_{\mathrm{f}}\left(1-E_{\mathrm{f}}\right)+\frac{E_{\mathrm{f}} B_{\mathrm{f}}}{1+\left(B_{\mathrm{f}} \alpha_{\mathrm{f}}\left(\beta^{0}, r^{0}, \delta_{\mathrm{f}}^{0}\right)\right)^{2}}\right] \cdot\left[\frac{\frac{L_{\mathrm{f}}}{\nu} \cos \beta^{0}}{1+\left(\frac{L_{\mathrm{f}}}{\nu} r^{0} \cos \beta^{0}\right)^{2}}\right] \\
& -L_{\mathrm{r}} D_{\mathrm{r}} \cos \left[C_{\mathrm{r}} \tan ^{-1}\left\{B_{\mathrm{r}}\left(1-E_{\mathrm{r}}\right) \alpha_{\mathrm{r}}\left(\beta^{0}, r^{0}\right)+E_{\mathrm{r}} \tan ^{-1}\left(B_{\mathrm{r}} \alpha_{\mathrm{r}}\left(\beta^{0}, r^{0}\right)\right)\right\}\right] \\
& \cdot\left[\frac{C_{\mathrm{r}}}{1+\left(B_{\mathrm{r}}\left(1-E_{\mathrm{r}}\right) \alpha_{\mathrm{r}}\left(\beta^{0}, r^{0}\right)+E_{\mathrm{r}} \tan ^{-1}\left(B_{\mathrm{r}} \alpha_{\mathrm{r}}\left(\beta^{0}, r^{0}\right)\right)\right)^{2}}\right] \\
& \left.\cdot\left[B_{\mathrm{r}}\left(1-E_{\mathrm{r}}\right)+\frac{E_{\mathrm{r}} B_{\mathrm{r}}}{1+\left(B_{\mathrm{r}} \alpha_{\mathrm{r}}\left(\beta^{0}, r^{0}\right)\right)^{2}}\right] \cdot\left[-\frac{\frac{L_{\mathrm{r}}}{\nu} \cos \beta^{0}}{1+\left(\frac{L_{\mathrm{r}}}{\nu} r^{0} \cos \beta^{0}\right)^{2}}\right]\right) \\
& g_{1}=\frac{1}{m \nu}\left(D_{\mathrm{f}} \cos \left[C_{\mathrm{f}} \tan ^{-1}\left\{B_{\mathrm{f}}\left(1-E_{\mathrm{f}}\right) \alpha_{\mathrm{f}}\left(\beta^{0}, r^{0}, \delta_{\mathrm{f}}^{0}\right)\right\}\right]\right. \\
& \cdot\left[\frac{C_{\mathrm{f}}}{1+\left(B_{\mathrm{f}}\left(1-E_{\mathrm{f}}\right) \alpha_{\mathrm{f}}\left(\beta^{0}, r^{0}, \delta_{\mathrm{f}}^{0}\right)+E_{\mathrm{f}} \tan ^{-1}\left(B_{\mathrm{f}} \alpha_{\mathrm{f}}\left(\beta^{0}, r^{0}, \delta_{\mathrm{f}}^{0}\right)\right)\right)^{2}}\right] \\
& \left.\cdot\left[-B_{\mathrm{f}}\left(1-E_{\mathrm{f}}\right)-\frac{E_{\mathrm{f}} B_{\mathrm{f}}}{1+\left(B_{\mathrm{f}} \alpha_{\mathrm{f}}\left(\beta^{0}, r^{0}, \delta_{\mathrm{f}}^{0}\right)\right)^{2}}\right]\right) \\
& g_{2}=\frac{L_{\mathrm{f}} \cos \beta}{I_{z}}\left(D_{\mathrm{f}} \cos \left[C_{\mathrm{f}} \tan ^{-1}\left\{B_{\mathrm{f}}\left(1-E_{\mathrm{f}}\right) \alpha_{\mathrm{f}}\left(\beta^{0}, r^{0}, \delta_{\mathrm{f}}^{0}\right)\right\}\right]\right. \\
& \cdot\left[\frac{C_{\mathrm{f}}}{1+\left(B_{\mathrm{f}}\left(1-E_{\mathrm{f}}\right) \alpha_{\mathrm{f}}\left(\beta^{0}, r^{0}, \delta_{\mathrm{f}}^{0}\right)+E_{\mathrm{f}} \tan ^{-1}\left(B_{\mathrm{f}} \alpha_{\mathrm{f}}\left(\beta^{0}, r^{0}, \delta_{\mathrm{f}}^{0}\right)\right)\right)^{2}}\right] \\
& \left.\cdot\left[-B_{\mathrm{f}}\left(1-E_{\mathrm{f}}\right)-\frac{E_{\mathrm{f}} B_{\mathrm{f}}}{1+\left(B_{\mathrm{f}} \alpha_{\mathrm{f}}\left(\beta^{0}, r^{0}, \delta_{\mathrm{f}}^{0}\right)\right)^{2}}\right]\right)
\end{aligned}
$$




\section{ACKNOWLEDGMENT}

The authors would like to thank the reviewers and the Associate Editor for their helpful and detailed comments, which have helped to improve the presentation of this paper.

\section{REFERENCES}

[1] A. Vahidi and A. Eskandarian, "Research advances in intelligent collision avoidance and adaptive cruise control," IEEE Trans. Intell. Transp. Syst., vol. 4, no. 3, pp. 143-153, Sep. 2003.

[2] L. Li, F. Y. Wang, and Q. Zhou, "Integrated longitudinal and lateral tire/ road friction modeling and monitoring for vehicle motion control," IEEE Trans. Intell. Transp. Syst., vol. 7, no. 1, pp. 1-19, Mar. 2006.

[3] J. Ackerman, J. Guldner, W. Sienel, R. Steinhauser, and V. I. Utkin, "Linear and nonlinear controller design for robust automatic steering," IEEE Trans. Control Syst. Technol., vol. 3, no. 1, pp. 132-143, Mar. 1995.

[4] H. Peng and M. Tomizuka, "Vehicle lateral control for highway automation," in Proc. Amer. Control Conf., San Diego, CA, May 23-25, 1990, pp. 788-794.

[5] H. Peng, T. Hessburg, M. Tomizuka, and W. Zhang, "A theoretical and experimental study on vehicle lateral control," in Proc. Amer. Control Conf., Chicago, IL, Jun. 24-26, 1992, pp. 1738-1742.

[6] E. Ono, S. Hosoe, H. D. Tuan, and S. Doi, "Bifurcation in vehicle dynamics and robust front wheel steering control," IEEE Trans. Control Syst. Technol., vol. 6, no. 3, pp. 412-420, May 1998.

[7] L. R. Ray, "Nonlinear state and tire force estimation for advanced vehicle control," IEEE Trans. Control Syst. Technol., vol. 3, no. 1, pp. 117-124, Mar. 1995.

[8] J. E. Naranjo, C. Gonzalez, R. Garcia, T. Pedro, and R. E. Haber, "Powersteering control architecture for automatic driving," IEEE Trans. Intell. Transp. Syst., vol. 6, no. 4, pp. 406-415, Dec. 2005.

[9] L. Beji and Y. Bestaoui, "Motion generation and adaptive control method of automated guided vehicles in road following," IEEE Trans. Intell. Transp. Syst., vol. 6, no. 1, pp. 113-123, Mar. 2005.

[10] N. Kopell and L. N. Howard, "Bifurcations and trajectories joining critical points," Adv. Math., vol. 18, pp. 306-358, 1975.

[11] E. H. Abed and J. H. Fu, "Local feedback stabilization and bifurcation control, II. Stationary bifurcation," Syst. Control Lett., vol. 8, no. 5, pp. 467-473, May 1987.

[12] J. Guckenheimer and P. Holmes, Nonlinear Oscillations, Dynamical Systems, and Bifurcations of Vector Fields. New York: Springer-Verlag, 1983.

[13] S. N. Chow and J. K. Hale, Methods of Bifurcation Theory. New York: Springer-Verlag, 1992.

[14] L. N. Howard, "Nonlinear oscillation," in Nonlinear Oscillations in Biology, F. C. Hoppendteadt, Ed. Providence, RI: Amer. Math. Soc., 1979, pp. 1-67.

[15] D.-C. Liaw and E. H. Abed, "Stabilization of tethered satellites during station-keeping," IEEE Trans. Autom. Control, vol. 35, no. 11, pp. 1186-1196, Nov. 1990.

[16] D.-C. Liaw and E. H. Abed, "Active control of compressor stall inception: A bifurcation-theoretic approach," Automatica, vol. 32, no. 1, pp. 109$115,1996$.

[17] D.-C. Liaw and C.-C. Song, "Analysis of longitudinal flight dynamics: A bifurcation-theoretic approach," J. Guid. Control Dyn., vol. 24, no. 1, pp. 109-116, 2001.

[18] D.-C. Liaw, K.-H. Fang, and C.-C. Song, "Bifurcation analysis of power systems with tap changer," in Proc. IEEE ICNSC, Tucson, AZ, Mar. 19-22, 2005, pp. 283-288.

[19] J. Y. Wong, Theory of Ground Vehicles. Hoboken, NJ: Wiley, 2001

[20] E. J. Doedel, "AUTO: A program for the automatic bifurcation analysis of autonomous systems," Congressus Numerantium, vol. 30, pp. 265-284, 1981.

[21] CarSim User Manual, Mech. Simul. Corp., Ann Arbor, MI, 2001, Version 5.11.

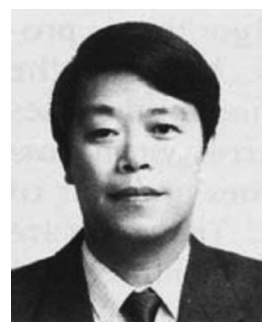

Der-Cherng Liaw (S'86-M'90-SM'02) received the B.S. degree in control engineering from National Chiao Tung University (NCTU), Hsinchu, Taiwan, R.O.C., in 1982, the M.S. degree in electrical engineering from National Taiwan University, Taipei, Taiwan, in 1985, and the Ph.D. degree in electrical engineering from the University of Maryland, College Park, in 1990.

During 1990-1991, he was a Postdoctoral Fellow with the Institute of Systems Research, University of Maryland. Since August 1991, he has been with the NCTU, Hsinchu, where he is currently a Professor of electrical and control engineering. His research interests include nonlinear control systems, spacecraft control, singular perturbation methods, bifurcation control, jet engine control, flight control, electric power systems and power electronics, radio frequency identification (RFID), and implementation issues.

Dr. Liaw served as a Designated Assistant to the Associate Editor for the IEEE TRANSACTIONS ON AUTOMATIC CONTROL during 1990-1992. He was also a member of Editorial Board of 1993 American Control Conference.

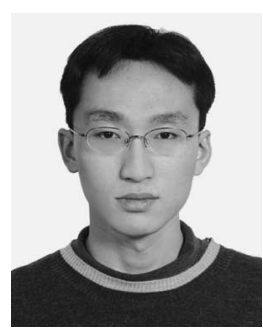

Hsin-Han Chiang (S'02-M'03) received the B.S. degree in electrical and control engineering from National Chiao Tung University (NCTU), Hsinchu, Taiwan, R.O.C., in 2001, where he is currently working toward the Ph.D. degree in electrical and control engineering.

His research interests include intelligent systems and control theory, fuzzy systems and control, automated vehicle control, and intelligent transportation systems.

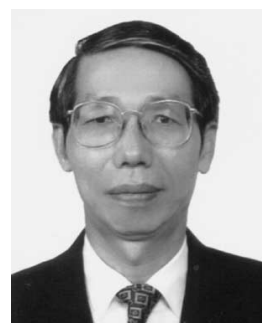

Tsu-Tian Lee (M'87-SM'89-F'97) was born in Taipei, Taiwan, R.O.C., in 1949. He received the B.S. degree in control engineering from the National Chiao Tung University (NCTU), Hsinchu, Taiwan, R.O.C., in 1970 and the M.S. and Ph.D. degrees in electrical engineering from the University of Oklahoma, Norman, in 1972 and 1975, respectively.

In 1975, he was appointed as an Associate Professor and, in 1978, as a Professor and Chairman of the Department of Control Engineering, NCTU. In 1981, he became a Professor and Director of the Institute of Control Engineering, NCTU. In 1986, he was a Visiting Professor and, in 1987, a Full Professor of electrical engineering with the University of Kentucky, Lexington. In 1990, he was a Professor and Chairman of the Department of Electrical Engineering, National Taiwan University of Science and Technology (NTUST), Taipei, Taiwan, R.O.C. In 1998, he became a Professor and Dean of the Office of Research and Development, NTUST. In 2000, he was appointed as a Chair Professor with the Department of Electrical and Control Engineering, NCTU. Since 2004, he has been with the National Taipei University of Technology, Taipei, where he is currently the President.

Prof. Lee was elected as a Fellow of the Institution of Electrical Engineers in 2000. He became a Fellow of the New York Academy of Sciences in 2002. He has served as a member of the Technical Program Committee and a member of the Advisory Committee for many IEEE sponsored international conferences. $\mathrm{He}$ is now the Vice President for Conferences of the IEEE Systems, Man, and Cybernetics Society. He received the Distinguished Research Award from the National Science Council, Taiwan, R.O.C., in 1991-1998; the Academic Achievement Award in Engineering and Applied Science from the Ministry of Education, Taiwan, R.O.C., in 1997; the National Endow Chair from the Ministry of Education, Taiwan, R.O.C., in 2003 and 2006, respectively; and the TECO Science and Technology Award from TECO Technology Foundation in 2003 . 